\title{
Infroductory Review
}

\section{The Study of Shape Transformation after D'Arcy Thompson}

\author{
FRED L. BOOKSTEIN \\ Center for Human Growth, Division of Biological Sciences, \\ and Society of Fellows, The University of Michigan, \\ Ann Arbor, Michigan 48104
}

Received 5 November 1976; revised 3 January 1977

Contents: I. Introduction, 717. II. The authentic Method. Thompson's Own Work, 178. Later Examples, 181. Difficulties, 186. III. Compromises. Analysis of Growth Gradients, 187. Simulations, 191. Vector Displacement Methods, 195. Multivariate Morphometrics, 198. IV. The method of Biorthogonal Grids. A Privileged Coordinate System, 200. Existence and Form of Biorthogonal Grids, 206. Sample Computations, 210. A Future for Transformation Analysis, 215. Bibliography, 217.

\section{INTRODUCTION}

The immense respect in which the biological profession holds D'Arcy W. Thompson's On Growth and Form is not explicable by the usual norms of scientific seminality. It is not that this work has founded a discipline, or solved long-standing puzzles crucial to progress elsewhere. Nor did Thompson's methodology change the face of a field. The direction of mathematical biology in this century has been away from the geometry he espoused, toward formal models from differential equations and information theory. If today there is such a thing as a "science of form" [17], its foundations derive from work half a century later than Thompson's. The sturdy popularity of this book resides rather in its own unique method, a quite non-Darwinian search for geometric simplicity which is classical in its mathematics, eclectic in its natural history, and perfect and elegant in its prose. Subsequent complication and refinement of the explanations does not affect our delight in the simple clarity of Thompson's models.

The most original theme of this whole work, and the most admired, has left the fewest scientific offspring. This is the chapter "On the Comparison of Related Forms" introducing the almost unprecedented method of transformations and their visualization by Cartesian grids. Of all such grids ever

(1) Elsevier North-Holland, Inc., 1977 
published, the majority, I believe, are still of Thompson's own penning, reprinted innumerable times. His is the only fundamental investigation of the problem, ubiquitous throughout the biological and medical sciences, of analyzing change of form. An author whose work touches on this theme will ordinarily quote a sentence or two of Thompson's text, express his despair of quantifying the diagrams, and then turn in the course of actual data analysis to simpler, inadequate models of less geometric insight but greater arithmetic tractability. Not for Thompson these compromises with "findings"! - - his concern was pure method. There is a greatness of scope about his original construction which invariably compels respect. Successive generations of methodologists, trying to alter the method or extend it slightly, have never built on each other's work, but only on Thompson's own, after the manner of conservatory students reconstructing Mozart's Requiem. There has sometimes been greater reward in a less ambitious task, the chipping off of small bits of the technique congruent with the computing technology of the time, as for allometry in the 1930s, multivariate morphometrics today. But all authors following so on Thompson's chapter acknowledge that they have not matched his scope or elegance, have not grasped even as well as he the puzzle of relating mathematics to change of form. Today Thompson's method of transformations faces an audience itself transformed in numbers, financial resources, and computing power; but the method remains as intractable as it seemed in 1917, and just as "promising," intriguing, and frustrating.

In this essay I shall summarize Thompson's own exposition and the few efforts to apply it directly to the analysis of biological shape change. I shall then review certain recurrent compromises, more or less successful, intended to bridge the divergence between the purity of the method and the causal and statistical complexity of real data. In the final section I shall present some recent work of my own which attempts, as others have done, to quantify the original intent, without compromise.

\section{THE AUTHENTIC METHOD}

\section{THOMPSON'S OWN WORK}

D'Arcy Thompson's method is this: to represent a change of one shape into another by the single mathematical object which is the map of one shape onto the other, and then to visualize this mathematical object.

The preceding chapters of his essay had explored the applications of mathematical insight and geometrical models to various empirical forms and manifestations; the subject of shape change, to which Thompson now turns, is just another abstractable aspect of form. The virtue of mathematics is, after all, "to eliminate and to discard; to keep the type in mind and leave the single case, with all its accidents, alone. ... [The] deformation of a 
complicated figure may be a phenomenon easy of comprehension, though the figure itself have to be left unanalyzed and undefined. This process of comparison, of recognizing in one form a definite permutation or deformation of another, apart altogether from a precise and adequate understanding of the original 'type' or standard of comparison, lies within the immediate province of mathematics..." [55, p. 271]. His intention here, strictly methodological, has two thrusts. The first is to continue his search for the basis of form in force. "[The transformed representation] once demonstrated, it will be a comparatively easy task (in all probability) to postulate the direction and magnitude of the force capable of effecting the required transformation" [55, p. 272]. This goal is now considered quite archaic, as causation is now universally considered to work through natural selection at the level of ontogeny [18, p. 27lf.]. But the other thrust is still cogent. Thompson sets two conditions: "that the form of the entire structure under investigation should be found to vary in a more or less uniform manner, after the fashion of an approximately homogeneous and isotropic body..., and that our structure vary in its entirety, or at least that independent variants' should be relatively few" [55, p. 274]. These postulates accord with his belief that correlation of characters is the rule, that constituent parts of an organism never can evolve quite independently. From this he draws a sweeping conclusion:

When the morphologist compares one animal with another, point by point or character by character, these are too of ten the mere outcome of artificial dissection and analysis. Rather is the living body one integral and indivisible whole, in which we cannot find, when we come to look for it, any strict dividing line even between the head and the body, the muscle and the tendon, the sinew and the bone. Characters which we have differentiated insist on integrating themselves again; and aspects of the organism are seen to be conjoined which only our mental analysis had put asunder. The co-ordinate diagram throws in to relief the integral solidarity of the organism, and enables us to see how simple a certain kind of correlation is which had been apt to seem a subtle and a complex thing.

But if, on the other hand, diverse and dissimilar fishes can be referred as a whole to identical functions of very different co-ordinate systems, this fact will of itself constitute a proof that variation has proceeded on definite and orderly lines, that a comprehensive 'law of growth' has pervaded the whole structure in its integrity, and that some more or less simple and recognisable system of forces has been in control. It will not only show how real and deep-seated is the phenomenon of 'correlation,' in regard to form, but it will also demonstrate the fact that a correlation which had seemed too complex for analysis or comprehension is, in many cases, capable of very simple graphical expression [55, pp. 275-276].

Abandoning, then, his search for physical causation, in view of the complexity of the situation, Thompson sets out on a "floating mathematics for morphology, unanchored for the time being to physical science, but capable of valid generalisation on its own level" [23, p. 579]. He contents himself with a large variety of examples drawn from all over the two living 
kingdoms. Of these, that analysis linking Diodon and Orthagoriscus (= Mola) is probably the most famous of all. His analysis is quite instructive. (I have changed his figure numbers to correspond to mine.)

Figure [1(a)] is a common, typical Diodon or porcupine-fish, and in Figure [1(b)] I have deformed its vertical co-ordinates into a system of concentric circles, and its horizontal co-ordinates into a system of curves which, approximately and provisionally, are made to resemble a system of hyperbolas. The old outline, transferred in its integrity to the new network, appears as a manifest representation of the closely allied, but very different looking, sunfish, Orthagoriscus mola. This is a particularly instructive case of deformation or transformation. It is true that, in a mathematical sense, it is not a perfectly satisfactory or perfectly regular deformation, for the system is no longer isogonal; but nevertheless, it is symmetrical to the eye, and obviously approaches to an isogonal system under certain conditions of friction or constraint. And as such it accounts, by one single integral transformation, for all the apparently separate and distinct external differences between the two fishes. It leaves the parts near to the origin of the system, the whole region of the head, the opercular orifice and the pectoral fin, practically unchanged in form, size and position; and it shows a greater and greater apparent modification of size and form as we pass from the origin towards the periphery of the system.

In a word, it is sufficient to account for the new and striking contour in all its essential details, of rounded body, exaggerated dorsal and ventral fins, and truncated tail [55, pp. 300-301].

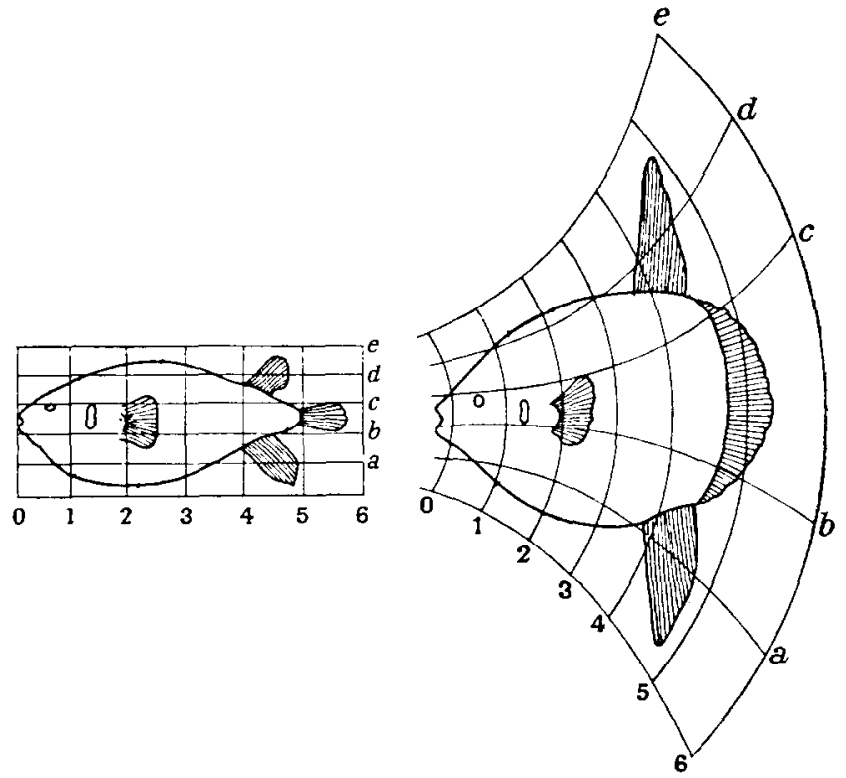

Fig. 1. Cartesian transformation from Diodon to Orthagoriscus (= Mola). From [55, p. 301]. 
A close reading of his own diagrams unearths several inconsistencies. The throat of Mola is the mirror-image of the area dorsal to the eye, but not so for Diodon; yet the grid is symmetrical, and hence has not accounted for this clear dorsoventral difference. The divergence of the outermost horizontal grid lines of Mola beginning at the far left, why does the divergence of the axes $b, c$ begin only behind the pectoral fin? The caudal margin of the anal fin has shifted between diagrams from axis $b$ to half way between axis $b$ and axis $a$, so that the vertical expansion of the axes rightward in Mola is insufficient to match the data. Should we reverse the roles of the two genera, so that Mola supported the rectilinear grid, what then would be the reading of the transformation? Would it still be made of conic sections, circles and hyperbolas? Thompson left no notes on the detailed construction of these drawings, so we cannot tell how he settled upon the axes that actually appear.

A subsidiary theme emerges warily in the course of Thompson's survey which has proved of most enduring interest among later students of the method. This is the diagrammatic scquencing of transforms and its cognate in paleontology, the search for intermediates. The most successful example is the very regular succession of deformations in the phylogeny of Equus, in which a form he declared dubious, Parahippus, is now considered not, after all, to belong. Thompson's interpolation is by drawing fractional parts of grid intersection displacements between figures. It is very simple, and takes no account of possible regional variation in evolutionary rate, but in this case it works beautifully. More famous than this is the less successful demonstration that the distortion from human to baboon differs "only in an increased intensity or degree of deformation" from the distortion from human to chimpanzee. His figures are assembled in my Fig. 2. "In both dimensions, as we pass from above downwards and from behind forwards, the corresponding areas of the network are seen to increase in a graduate and approximate logarithmic order in the lower as compared with the higher type of skull; and, in short, it becomes at once manifest that the modifications of jaws, brain-case, and the regions between are all portions of one continuous and integral process" [55, pp. 319-320]. It is unfortunate that Thompson inexplicably began with a "human skull" of a braincase impossibly large; in addition there are errors of drawing similar to those for the Diodon example, for instance the wandering of the gonial angle relative to the lower end of coordinate line 4. Thompson hoped to fill in this series too with intermediates, but the necessary fossil material was unearthed only after he ceased work. Without saying quite what he means by a "direct line of deformation," he concedes that neither of the apes lies "precisely" in the sequence of the other's hypothetical connection with man.

It seems that Thompson's postulate of homogeneity took precedence over his draftsmanship in most of these examples, and it is not surprising 
that grids published after him, with the exception of Needham's (Fig. 5 below), are invariably less legible.

\section{LATER EXAMPLES}

It is fair to say that, after Thompson's original publication, the method we are discussing underwent "vicissitudes" rather than "development." It has mainly been the last theme, interrelating the grid method with techniques of ordination, that accounts for most of the true grids computed by persons other than Thompson. A good review of this literature to the early 1950s may be found in [42]. No new examples at all appeared until the 1930s, perhaps owing to Thompson's failure to provide instructions. Colbert, in 1935, displayed a "Cartesian coordinate chart to illustrate the manner in which the skull of Rhinoceros might have evolved through Gaindatherium from a primitive form such as Caenopus. ... This is essentially the method used so widely by D'Arcy Thompson, but here it is applied in a more detailed manner than was done by that author" [10]. The drawings are excellent, but the accompanying text runs only ten lines. Colbert seems to have come to no useful conclusions at all for his pains.

A major difficulty for Thompson in his drawings was the location of grid lines traversing large regions without landmarks, such as the cranial vaults in Fig. 2. A clever solution to this problem was put forward by Avery [1] in 1933. He inked a square grid on a small tobacco leaf and photographed it over its subsequent development. There resulted an empirical sequence of true Thompson transformations, from which he extracted directly the areas of the little grid quadrilaterals and turned the whole into an analysis of areal growth gradients after the fashion of Huxley.

Much more thoroughgoing in its use of geometry is a subsequent reanalysis of these data by Richards and Kavanagh, [43]. The leaf images, the points followed individually throughout the growth, are exact enough to support a differentiation everywhere. From the derivative one may ascertain just what growth there is in any direction, and the authors present the directions of maximum and minimum growth by little crossed axes, of length proportional to the directional growth rate, scattered over the leaf figures. It is not clear to me how these were computed, as the derivatives postulated by their model need to be estimated with delicacy whenever the current system is not Cartesian, which is to say, at all times after the instant of the tattooing. Tobler [55, Fig. 2] has recomputed these axes, still using Avery's original data, and shows unsystematic errors in Richards and Kavanagh's computations scattered throughout the images.

Criticism of the arithmetic should not obscure the advance those authors were explicitly attempting toward a tractable transformation theory. They consider their method a combination of the Cartesian transform with the method of growth gradients to provide both numerical and geometric 


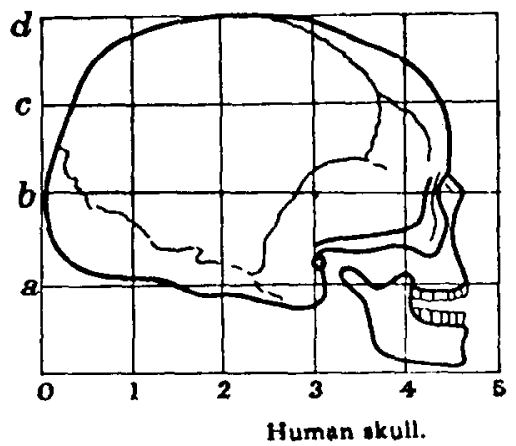

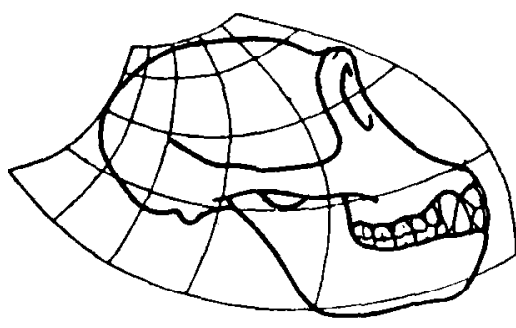

Skull of chimpanzee.

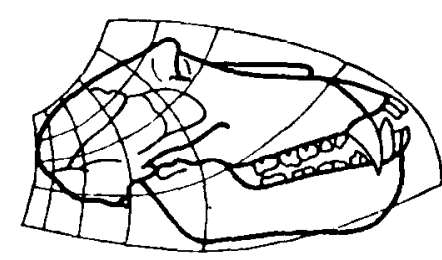

Skull of baboon.

FIG. 2. Cartesian transformation from "human skull" to chimpanzee and to baboon. From [55, pp. 318-319].

insight into observed processes. Their conclusion, in particular, is quite Thompsonian in spirit:

It seems reasonable to expect that the pattern of specific growth-rates should be explicable in terms of the characteristics of the growing material at the various points in the organism. ...In the example of the tobacco leaf, there was a tendency for the direction of maximum rate to coincide with the direction of the vascular bundles. It may be worthwhile, in future investigations, to see how closely the directions of these maximum and minimum rates can be related to recognizable structural characteristics of the organisms.

In cases in which the directions of the extreme rates prove to be tied in this manner to definite structures within the organism, the problem of describing the growth transformation becomes somewhat more definite. At any instant the directions of extreme rates determine throughout the organism three families of surfaces such that any member of one family intersects any member of the other families at right angles. (The simplest example of such a system of surfaces is, of course, the set of planes determining a rectangular coordinate system. In the case of growth in a plane, the directions of extreme 
growth-rate determine two mutually orthogonal sets of curves.) As the growth continues, these surfaces, though transformed, will still remain at right angles to each other [43, pp. 228. 229].

There were never any "future investigations," and these facts had to be rediscovered by me in the course of my own researches (see Sec. IV). The geometry of this analysis has been imitated only once, and then only with the abandonment of the original impetus, the dependent variable having been converted from a shape change to a field of scalar growth rates. Erickson [15], demonstrating a computerized version of the calculations, neither makes any use of the directions of growth (only of its directionality, a scalar) nor cites Thompson in his references.

Other studies, unable to deal with descriptions of single grids in extenso, have explored the ordination parameter in Thompson's seriation scheme. Kummer [28] attempted a consistent reconstruction of the hominid line from Proconsul through modern man, then fitted many intermediate fossils to fractional positions along this transformation. The brilliance of the drawing is tempered somewhat by the goodness of fit to Piltdown Man and by his evident disdain for the possibility of regionally varying rates of hominization. He nowhere explains how he estimates the serial position of particular forms, nor why the distances all happen to be integral multiples of one-eighth. Lull and Gray [29], also using Thompson's device for interpolation linearized upon the boundary, find the coordinate method too sophisticated for the available data (which, for ceratopsians, are quite widely spaced). Their extrapolations buckle the plane, growth gradients become apparent too soon, and the transforms of ontogeny are not related to those of phylogeny in any comprehensible way. They conclude that nothing new about ceratopsian phylogeny can be discovered by this method, which ought not to replace the traditional system of "comparing single pairs of measurements." Yet they conclude, oddly, that the fault is in the data, not in the method: the Thompsonian grid is too attractive to be rejected.

Several studies have attempted to resolve the perplexities of the grid method by the application of some other statistical technique. In 1967 P. H. A. Sneath [51] published a method which, though called "trend surface analysis of transformation grids," is in fact a somewhat different product for the analysis of displacement trend-surface grids. Sneath's interest is not in the geometric features of the grids themselves, but only in the "factors underlying the deformation" and particularly in the gross differences in general shape. His purpose is taxonomic, not biotheoretic: he wishes taxonomists not to be fooled by repetitious correlated expansions that could be characterized by a few coefficients of a function. He borrows from geology the notion of a trend surface, which summarizes scalar data distributed over a map. Operating from an arbitrary sample of correspond- 

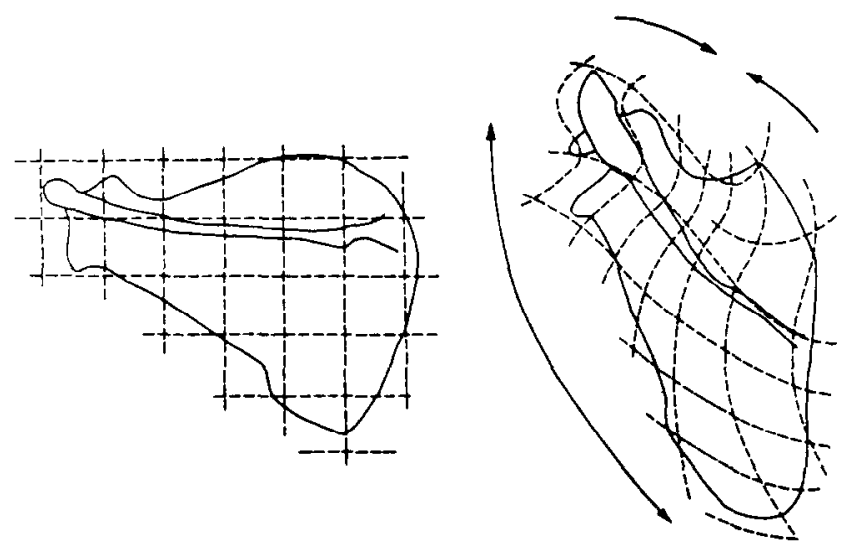

FIG. 3. Cartesian transformation from scapula of Papio to scapula of Gorilla, with indication of "cranio-lateral twist." From [40, Fig. 35]. Copyright 1973 by the University of Chicago.

ing points in pairs of images optimally superimposed, he fits separate trends for the two Cartesian displacements, vertical and horizontal, of the points in one diagram relative to their mates in another. He then simply partitions the trends into polynomial components and compares coefficients from fit to fit, for his intent is not, after all, the mapping of shape change. In spite of its basis in spatial data, this technique has no specific geometric or biological content. Components of the trend (i.e., quadratic, cubic,..., terms) link together discrepant curves and bends throughout the observed image, in disregard of biological structure. All local phenomena are smoothed out; in particular, there is no way of estimating growth rates in the various parts of the image. We are unable to return from the statistical manipulations to the original direct transforms of shape.

C. E. Oxnard [40, pp. 62-66] curiously reverses this tactic. He uses transformation grids to summarize some canonical axes produced by a conventional multivariate analysis. Having extracted three main factors discriminating primates by their scapulae, he selects three pairs of scapulae which differ only on a single score-first, second, or third. He draws the resulting transformations and looks for stresses which correspond to variables heavily loading on the factors. One grid is a "cranio-lateral twist," as in Fig. 3; another is a "mediolateral compression," and the third a "craniolateral stretching." Since factor scores are interval measures, it would be very fine if these categories of deformation were also. Can we ask how much cranio-lateral twist is in the transformation shown, and can we verify that reversing the direction of the transformation gives us the inverse amount of 
depicted twist? It would be very exciting if the transforms added or multiplied somehow to account for comparison of scapulae differing on all the scores. But as published, the words correspond to no formal properties and do not suggest any possibilities for measurement.

\section{DIFFICULTIES}

Thus the vicissitudes of the explicit method of transformation grids since its inception. Those who have attempted to make of the technique a tool for analysis or understanding commensurate with the elegance of its illustrations have of ten superimposed some arithmetic field over the original design for the engridment. Under this rubric I would include the elemental growth rates of those who studied Avery's leaf, and also the Cartesian displacements of Sneath. The others, those who did not add quantity to Thompson's original diagrammatic scheme, generally have failed to gain any geometric insight either, however carefully drawn their diagrams. I include Thompson himself in this latter category. In all his analyses, he rests with the possibility of a single system of forces-not a very well-defined analytical category. His goal was not to measure; he was content to exemplify the geometry logically prior to any measure.

The problem here is fundamental. It seems impossible to extract quantity from the Cartesian grid, as Thompson formulated it, in any straightforward way. Even after a decade during which the brightest graduate students all have had access to computing power adequate for large multivariate data sets, there is no hint in the literature of a line of attack upon quantification once one has painstakingly drawn out the Cartesian grid. Sokal and Sneath [52, pp. 82-83] argue from two quite well-drawn examples of intentionally incomprehensible accurate diagrams that the problem is one of feature enumeration. For any "realistic" grid fitting the data more closely than Thompson's (which is not a difficult accomplishment), various ebbs and flows of the lines become apparent; it is clear that more than one source of curvilinearity is at work, and these probably betoken a multiplicity of sources of variation. We have no visual facility to count these elementary "fields" or sense how they could be separated and measured. In the effort to talk about what is there we open our mouths and become speechless. There are stretches in certain slowly varying directions, and certain subtle changes of angle overall, and a constant shifting in the relative spacing of grid lines in both directions. The visual complexity of these grids is frustrating and indescribable, like distortions in an unflat mirror whose shape we cannot comprehend. Figure 4 exemplifies all these difficulties.

This is what Medawar meant when he called the method of transformations "analytically unwieldy" [31, p. 231]: that the transformations cannot be analyzed, broken into parts. It is very well to declare that a single shape change is all of a piece, but if families of them have to be analyzed, then we need some means of deciding when a whole collection is likewise all of a 


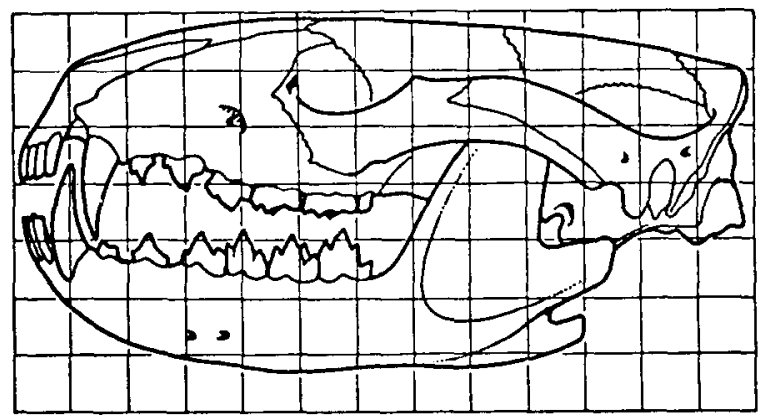

OASTURUS

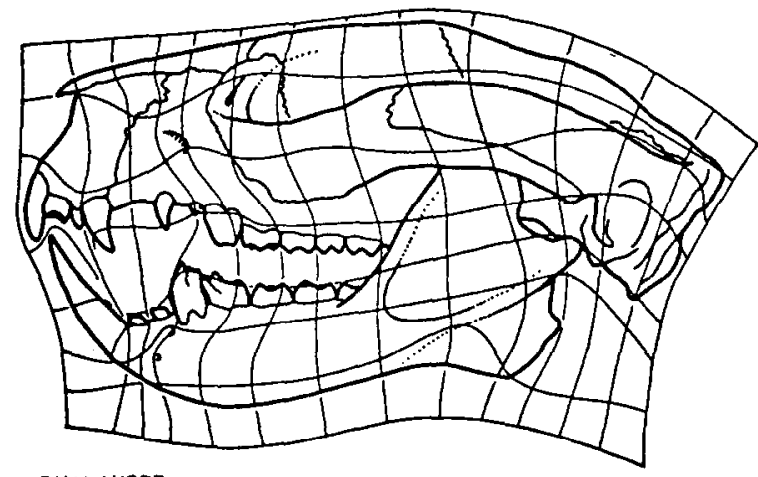

PHALANGER

Fig. 4. Example of a realistically drawn Cartesian transformation. From [52, Fig. 5-2]. W. H. Freeman and Company. Copyright @ 1963.

piece. To do this we need to count the pieces of pattern. When the data are as complex as for Lull and Gray's ceratopsians, the methods which succeed elegantly for the equids fail utterly. Where are we to turn?

The compromises I will review in the next section all determine in advance of the analysis certain distances or separations from whose statistical manipulation a grasp of structure is expected to emerge. It seems to me that no such method can be general, can be validly applied to more than a handful of data sets. I will argue cases as I explain them, only to conclude that we have to start over.

\section{COMPROMISES}

\section{ANALYSIS OF GROWTH GRADIENTS}

In a great many samples of related shapes, pairs of distance separations (as other types of variables as well) obey an equation of the form $y=b x^{k}$, 
the allometric equation, which is to say that they fall on a straight line in log-log plots. Such behavior will obtain whenever the intrinsic growth rates $(d x / d t) / x,(d y / d t) / y$ of the two separations are in constant ratio. In the special case in which one of the variates is general body size (measured by overall length), we are studying the change of proportions with size. Gould [16] would have this be the definition of allometry, irrespective of constancy of coefficients; but it is better for a discussion of Thompson to hew to Huxley's original definition (elaborated in [24]) in terms of numerically constant or slowly changing differential growth ratios among diverse parts and dimensions.

When a large number of short segments throughout a collection of organisms show constancy of pairwise relative growth rates during development, Huxley would have us speak of "the general distribution of growth potential," or growth gradients, which are the various coefficients $k$, growth rates with respect to some standard rate, distributed spatially over the organism. When these ratios are not quite constant, but vary smoothly with position and biological age, we may still compute the gradient field as it varies over successive growth periods.

In his original publication, Huxley interpreted his method as a quantitative refinement of Thompson's original scheme. Referring to the Diodon transform, Fig. 1, he writes:

If, as D'Arcy Thompson points out, the transformation, so difficult to understand at first sight, becomes readily comprehensible on the idea of an orderly change in the distribution of growth-activity along the axis of the body, then clearly the proportions of the animal must be continually changing so long as it is increasing in absolute size, or at least over a long space of time. But the fish's outline and the system of co-ordinates drawn to fit it represent the state of affairs only at one particular moment of its life-history. If the fish had grown to twice the bulk, its proportions would have changed, and the co-ordinate grid would have to be altercd; yet the underlying growth-gradient might have remained wholly unaltered. ...

For this reason, the co-ordinate method, while of the utmost importance as affording a graphic and immediate proof of the need for postulating regularities in the distribution of growth throughout the body, is of little use for detailed analysis, because by its nature it neglects the fundamental attribute of differential growth, namely the change of relative proportions with absolute size: it is static instead of dynamic, and substitutes the short cut of a geometrical solution for the more complex realities actually underlying biological transformation [24, pp. 105-106].

Apparently Huxley never noticed what emerges from four decades' hindsight: the method of gradients requires that the directions in which the gradient is likely to go be specified in advance-a "geometrical" input. The reason for this is essentially algebraic. Huxley knew that if separate parts of a limb manifest a nontrivial growth gradient, then the whole length is necessarily in exact allometric relation with none of its parts. He did not notice a simpler, geometric difficulty. It growth proceeds allometrically with different coefficients in two different directions from the same point, then 
even though the total area is growing allometrically, displacements in arbitrary directions are not. A rectangle growing as $x^{m}$ along one side and as $x^{n}$ along the other will have a diagonal growing as $\sqrt{x^{2 m}+x^{2 n}}$, which is not in allometric relationship with either side. I have already discussed the modification of this system by Richards and Kavanagh to account for directionality; the result is still expressed in terms of scalar fields-rate of increase in area, anisotropy-not leading to any major advance in the praxis of data analysis.

In practice, then, the method of growth gradients has been applied only when the axes along which gradients are to be measured are constant and fixed in advance. In the commonest instance, there is only one dimension of extent to be had, so that the gradient is necessarily a function of one real variable, a particularly simple sort. A locus classicus of this style is the work on segmental lengths of arthropod limbs [24, 36, 37, 54]. Medawar [30] simplified the algebraic treatment of these unidimensional gradients by introducing an explicit function for the relative position of any landmark as a function of age. (Yates [60] showed by a reanalysis that Medawar's data, human vertical proportions, do not support the verbal interpretation he placed upon them; but this is not the fault of the formal innovation.) For systems without persevering landmarks, such as growing plant tissues, the necessary mathematics is more complex: the age of the specific tissue must be entered as an additional variable. Salamon, List, and Grenetz [47] exemplify this more sophisticated analysis for the "streak photograph," a data-collection device which represents differentials explicitly by divergence of neighboring streaks.

In systems of more than one intrinsic spatial dimension, there are two methodological possibilities. In the study of accretionary growth, growth localized at a growing edge, it is common to measure separation along axes which vary with the developmental stage of the organism in some natural fashion. Moss and Salentijn [35], believing the growth of the human mandible to he of this form, measure distances along a certain logarithmic spiral which includes three mandibular foramina. Raup [41] models the snail shell by a basic plane section simultaneously rotating about an axis, moving along that axis, and increasing in scale. Shiells [50] measures a growing shell along its circumference.

A second possibility is the provenance of a specialized multidirectional coordinate system with symmetry properties. Needham [38] used the principal body axis and the abdominal segmental boundaries normal to it to derive a true two-dimensional growth gradient for the crab Pinnotheres pisum. Figure 5 summarizes his quantification in a remarkable Cartesian transformation grid much clearer than a contoured plot of directional $k$ 's could be. Such an analysis should not be confused with similar analyses of two-dimensional gradient fields in one direction only, as in Needham [39, Fig. 4.6], or of one-dimensional gradient fields followed over successive 

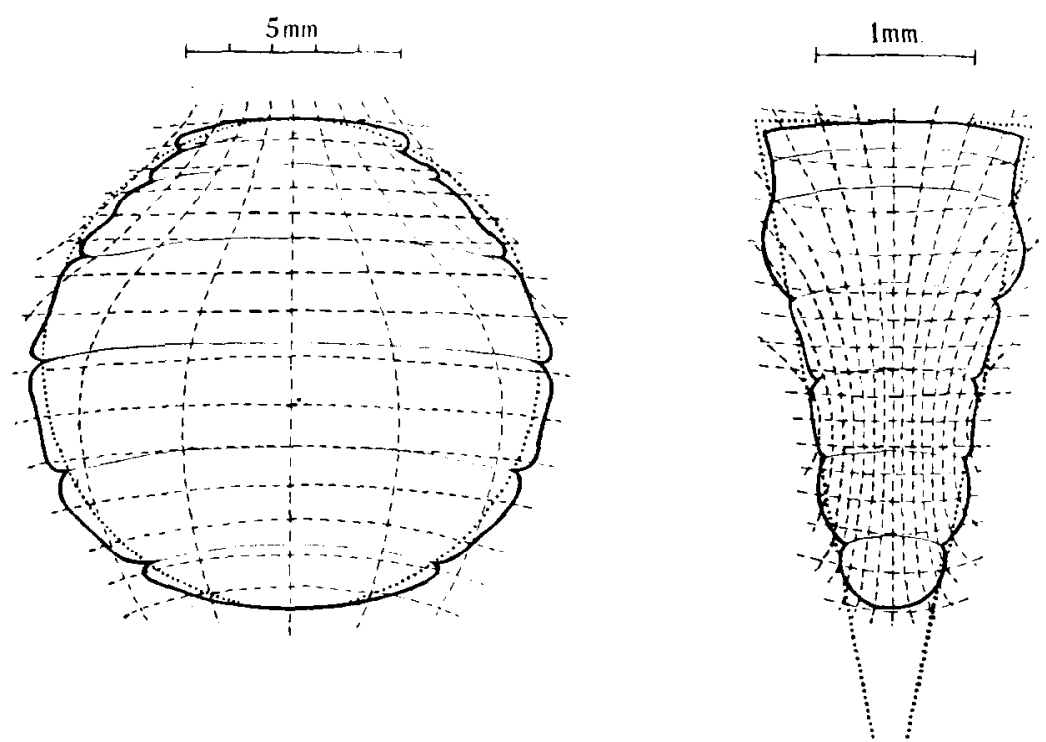

FIG. 5. Cartesian coordinate transformations between the adult female (A) and the adult male (B) of Pinnotheres pisum. Each grid is the trace of a grid squared upon the other form. The transformation is from a near-triangle to a near-circle. From [38, Fig. 5]. Reproduced by permission of the Royal Society.

stages, as in Needham [36], Blackith, Davies, and Moy [2], or Brown and Davies [7]. It is very difficult to combine gradients and grid in the same diagram. The illustrations of Ambystoma larvae in Richards and Riley [44], for instance, manifest far too many straight lines to suggest any Thompsonian homogeneity of deformation.

Akin to symmetry in space is constancy over time: systems in a "steady state" of growth. For instance, there is a considerable botanical literature on the subject of shoot and root meristems, centers of morphogenetic patterning which lie in invariant spatial relation to the steadily differentiating mass of tissue produced in their past. Schüepp [48] places upon sections of meristems coordinate curves which are trajectories, relative to the growth center, of particular cells as the continued growth of the meristem pushes it away from them. In the resulting diagrams, any two regions each bounded by a crosscut of those trajectories at points of constant age are true Thompson transforms of each other, for they correspond point for point inside. In such stable growth patterns, time is an ignorable coordinate. Any future snapshot is identical to the present snapshot with the points systematically relabeled. Certain spacings along those trajectories in any sec- 
tion then correspond to specific local growth rates across sections. A broad geometric theory of meristematic activity making extensive use of this particular sort of symmetry may be found in [49].

Both these extensions of gradient analysis evade Thompson's demand that the dependent variable should be form itself. A method appropriate for the study of segment series cannot be modified to analyze vertebrates: the higher class has no natural segment boundaries along which to align the vector separations to be measured. These boundaries are perpendicular to a growth gradient along a principal body axis, but in general shape changes there is no such axis (away from the midline) for correlating directions at finite distance. Distances measured today along a straightedge will correspond to arcs more or less curved tomorrow [55, p. 320], but the proponents of growth-gradient analysis do not instruct us in which arcs to use. Somehow the features of form - the bulges and bends, convexities and concavities and protuberances-are lost in the reduction to quantity. This will not do: there is not enough geometry left.

\section{SIMULATIONS}

Under this head I choose to list three analyses unknown to each other which I find to share a common spirit closer to Thompson's. Each one investigates growth rates in the small throughout an organ, and then discusses the extent to which the separate local changes cohere in the global change of form actually observed. Always the data are not capable of explicitly supporting the leap to larger patterns, creating a logical gap which is bridged by mathematical assumptions that simulate the final change of form.

P. B. Green [20] studies the patterns of surface growth which might account for a particular observed invariance of shape in three dimensions. His subject is the apical tip of Nitella, which maintains its shape-a hemisphere surmounting a cylinder-as it incorporates new plasm and grows upward. It is a stubborn mathematical fact that the preservation of shape does not determine a unique local growth-rate field. Anisotropy is optional; it need merely be properly coordinated with the change of directional gradients over a curving surface. For radially symmetric systems such as the Nitella tip, alternative growth models can all be expressed as functions of meridional distance from the apex. Different functions correspond to quite different local mechanisms, for while isotropic growth suggests scalar morphogenetic fields, anisotropic growth requires a histological asymmetry grounded in some cytoplasmic texture. A certain mathematical elegance is associated with this analysis, as form invariance leads to a differential equation directly governing the rate function. The calculus is developed further by da Riva Ricci and Kendrick [46]. To settle 
the question of isotropy Green proceeded to measure actual rates of increase of separation between points marked with microspheres on the growing hemispherical surface. He finds, in fact, anisotropies which reverse their sense over cell age. The origin of directionality in the known structures of plant cell walls is reviewed by Green [21]. Such a strategy, despite its clegance, cannot be expected to generalize to systems lacking the peculiar symmetries of this organ (radial symmetry and invariance of form over time), as the necessary differential equation can no longer be produced.

The anatomist D. H. Enlow has long studied the details of human bone growth. His findings regarding the craniofacial region are collected in [13, 14]. Bone grows generally by "remodeling," unending deposition and resorption. These alternatives can be discriminated in photomicrographs. Enlow has carefully examined the nature of growth on every surface, across every tuberosity, of the normal craniofacies. He is thereby able to specify, for any particular anatomical form of interest, what local growth processes, taken in concert, are responsible for the observed changes of form and relative position. There are certain recurrent themes, for instance that $\mathrm{V}$-shaped forms generally grow by deposition on the inside of the $\mathrm{V}$ and resorption on the outside, and thus displace themselves as they grow.

From his analysis, he concludes that form moves through bony tissue, slowly changing under the impulse of external correlations and alignments. There is an insight here which goes beyond Thompson. Growth may remove material as easily as it deposits it. Shape is in part negative, adjustment to space outside the material boundary. In fact, in a growing bone maintaining a shape which is not convex, certain surfaces are always necessarily shrinking, undergoing "decretionary" growth. In addition, within the face as a whole, bones are moved passively by abutting on other bones themselves growing. In principle such a system for analyzing growth should account for all changes of bone shape, abnormal as well as normal, in terms of the two basic processes, deposition and resorption, all over the growing surface. Unfortunately the quality of present numerical data is insufficient to support quantitative analysis systematizing the detailed qualitative and directional analysis. We do not know how to talk clearly about widely spaced simultaneous remodeling processes which together very nearly preserve a functional form. If a geometric formalism could be invented to represent usefully the elegant osteology of this method, analysis could advance rapidly beyond the image of Fig. 6 , and perhaps I might no longer consider this work a "compromise" with Thompson.

My last example of simulation is an intensive study of one phase of newt neurulation [25]. The data are a scalar field of cell size decrease from stage 13 to stage 15 of the embryo, together with measures of the notochord's exogenous extension during that period. The analysis produces, according to a mathematical model of tissue shear, a predicted shape change for the 


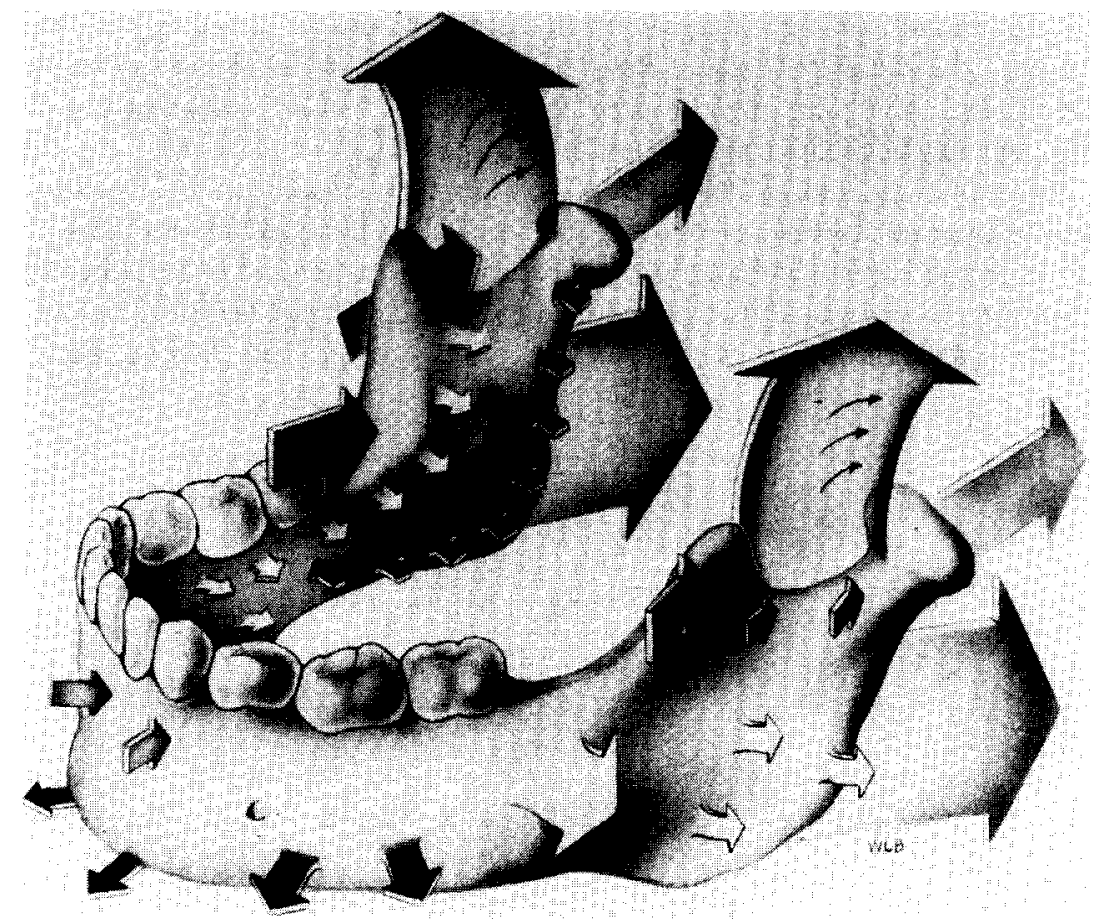

Fig. 6. Summary diagram of the growth of the mandible. Resorption is indicated by arrows pointing in, deposition by arrows pointing out. From [14, Fig. 3-150]. Reproduced by permission.

whole neural plate. In the form of a summary Cartesian transformation grid (Fig. 7), this simulation may be compared with an observed grid previously published: it is a fair replica. The most interesting feature of the simulation is the isolation of anisotropy. The separate cells of stage 13 shrink in cross-section individually and isotropically, whereas the extension of the notochord is highly directional. The authors put forward a rather complicated model for the propagation of this shear through the bulk of the tissue by rearrangement of cell-cell connections. At present this phase of the simulation has an irreducibly stochastic component, which I trust can be removed by further mathematical analysis. But it is not necessary to believe in the reality of this mechanism to appreciate its elegance and parsimony. In differential growth analysis over areas, one consequence of anisotropy is an increase in the number of essential numerical parameters from one to three (two growth rates, or areal growth rate and anisutropy, together with orientation of the principal axis). The Jacobson-Gordon model shows that over most of a tissue one can make do with the scalar representation alone 
if the more complex specification of anisotropy is present as a driving force abutting the simpler system. Their conclusion is quite in the spirit of Thompson: "the joint operation of two physical forces is necessary and sufficient to effect this transformation" [25, p. 191].

None of these simulations presently are capable of mathematical generalization to transformation grids in their full complexity. Each contains at least one good idea which ought to be present in any more inclusive scheme. From Green, the nugget is the treatment of surface elements; from Enlow, the idea of simultaneous positive and negative growth; from Jacobson and
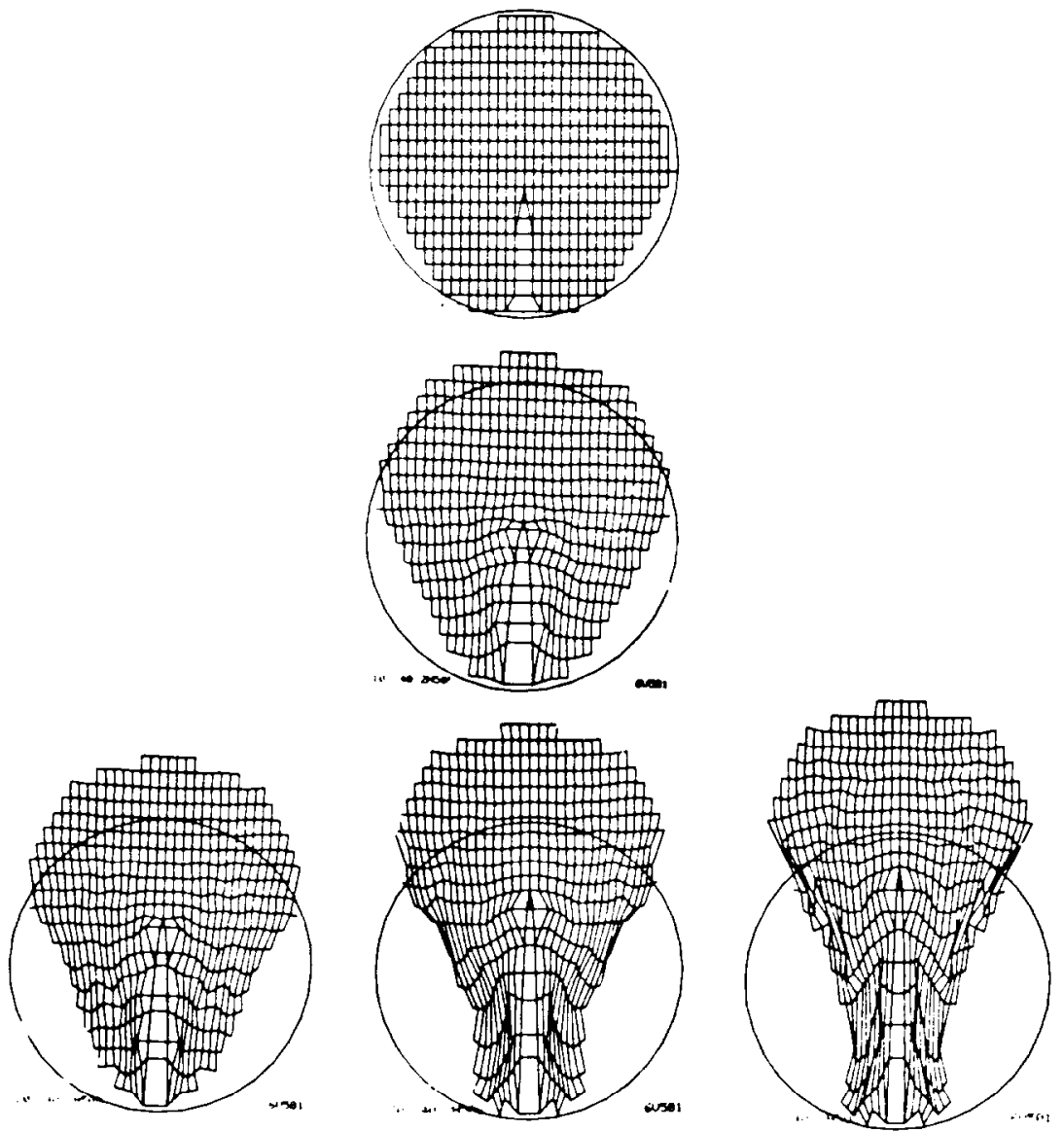

FIG. 7. Cartesian transformation grid for the simulation of neural plate development. The wedge in lower center is the developing notochord. The particular computer run depicted here allowed shear but no isotropic shrinkage of cell areas; it simulates the shape change from disk to keyhole but not the relative proportions of top and bottom. From. [25, Fig. 16]. Reproduced by permission. 
Gordon, the modeling of anisotropy as viscous response to exogenous shear. All are suggestive, but none are yet generally satisfactory.

\section{VECTOR-DISPLACEMENT METHODS}

A large body of work directly relevant to our theme arises from the problems faced by the practicing orthodontist. He is in the business of manipulating a future shape, which in these pages will be considered to be represented by a sagittal cephalogram, an $x$-ray of the head from the side. To prescribe a treatment he must first predict a plausible outcome in the absence of manipulation. The predicted mature shape may be thought of as a geometric transformation applied to the growing form at any stage; the transformation we need will presumably average somehow the specific changes in appropriate populations followed over time. Furthermore, any particular face is more or less distinguished from an aesthetically ideal shape; to characterize the transformation between these two is to provide a hint of how the orthodontist might restore grace and balance.

In view of this multiplicity of practical motivations, it is surprising that the techniques of the field are mainly variations of a single limited theme. In all methods known to me, with the exception of Enlow's, the observed shape is at some point strictly superimposed on a normal shape or on an earlier shape from the same ontogeny. The pair of shapes are then related by the vector displacements from image to image of all the structures the investigator cares to follow. In the course of the analysis there must necessarily be an algorithm for registering and orienting the images. These rules are arbitrary and vary from analysis to analysis; and unfortunately they affect all quantitative conclusions.

C. F. A. Moorrees [33, 34] constructs transformation grids explicitly. His registration is rather peculiar. The nasion has two fixed coordinates; the sella turcica and anterior nasal spine, one each. The horizontal and vertical axes are scaled separately, and oriented according to "natural head position". The actual grid, which may be drawn by hand or by computer, is drawn separately for the upper and the lower face, with a discontinuity along the line of occlusion. Figure 8 is an example of the resulting diagram. Having produced a sort of transformation grid, Moorrees and his colleagues do not really know what to do with it. In fact, the complex registration has made it statistically intractable. The grid is but a picture of a certain idiosyncratically standardized collection of vector displacements. The original biological form has been altered in the engridment, because of the separate scalings in two directions. The grid as drawn is not the transformation to the observed form from normative configuration or past form, for a new anisotropy has been imposed. Also by virtue of the registration, certain points of the grid are intrinsically less variable than others. Because of such constraints, Moorrees's work, whatever its contribution to orthodontic diag- 


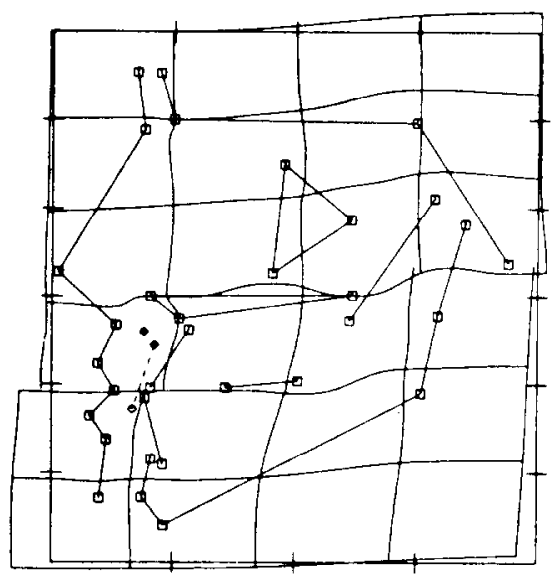

FIG. 8. Cartesian pseudogrid for a normalized cephalogram indicating "marked mandibular prognathism and cephalad position of sella turcica, basion, articulare, and gonion." The facial profile is to the left. From [34, Fig. 5B left]. Copyright $\odot$ International Orthodontic Congress.

nosis, cannot help us in the mathematical development of Thompson's original intent. His grids are simply a visual summary of a complicated spatial correspondence.

Several other traditions reduce these constraints to the minimum necessary, two: one registration (meaning point superposition) and one orientation (meaning line alignment). (One occasionally finds a technique of two point superpositions, but never, to my knowledge, two alignments.) Walker and Kowalski $[58,59]$ register on the sella turcica and use for horizontal the line from the base of the occipital bone to the center of the palate. Several systems, reviewed in Merow [32], register on the sella but use the Frankfort horizontal, the tangent to the top of the porion which passes through the orbitale. Delattre and Fenart [12] register on the vestibulary apparatus and its implied horizontal. Ricketts [45] registers the mandible on the pogonion and a special circular arc. Superposition according to any of these customs produces a series of vectors representing for any landmark its succession of coordinates in this series of coordinate systems, and thereby, implicitly, a succession of Cartesian grids. They have all this common flaw: the variable pictured is not what we need. The method of transformations has no role for fixed points or orientations; it is couched in terms of how points grow apart from each other, not from some arbitrary center. The import of a diagram such as Fig. 9 is wholly misleading. For any of the vector-displacement techniques, statistical treatment is extraordinarily problematical. All location measures are beset by the measurement error of the standard location and orientation in addition to their own. This supplies a functional 
correlation between any pair of displacement measurement errors which is of constantly changing magnitude and direction. Points near the center of registration move less than points farther away, and all points generally move away from the fixed point-both these trends anatomically quite meaningless. Landmarks near each other move in roughly parallel fashion, as the divergence between them is swamped by the joint susceptibility to pushes outward by all tissues between them and the fixed point. When one bit of tissue undergoes a small unconformity of development, all landmarks on the far side of it manifest the same jog in their traces. Then the structure of variation of the outermost vectors is a completely opaque composite of variations there and elsewhere; to untangle it one has to start over by other procedures entirely. And one must disentangle thus, for conclusions about growth which vary with the registration one chances to use are worse than no conclusions at all.

With all their flaws, the registered grids have one great temporary advantage. They remove just enough of the "floating" quality of Thompson's general framework to make possible a multivariate statistical analysis, however flawed. Vectors and displacements have population averages and

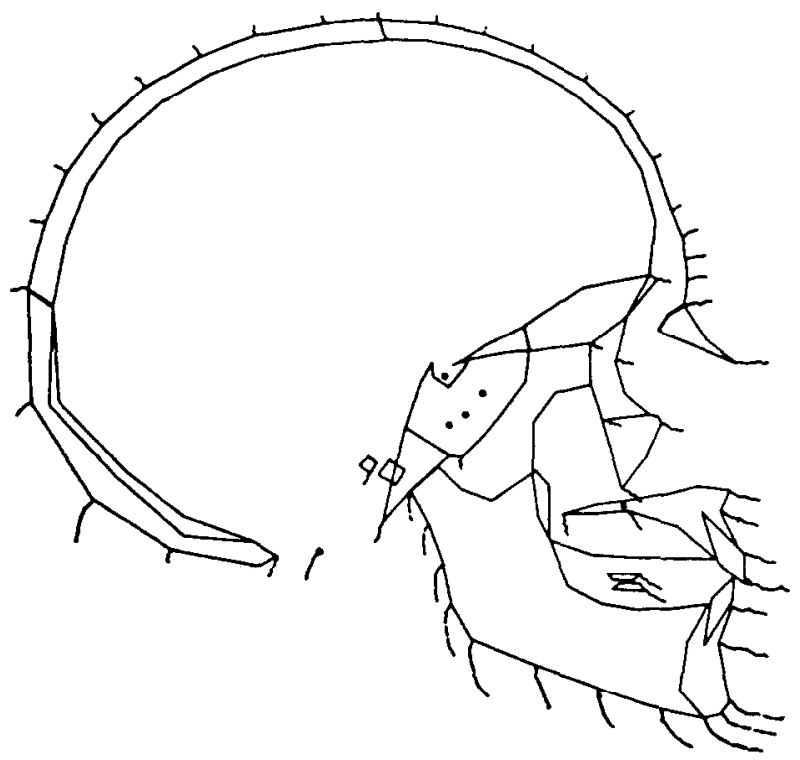

Fig. 9. Vector-displacement diagram for the progression of various landmarks from a starting configuration. The whiskerlike lines each follow one landmark through successive outlines, none of which but the first are drawn. Note the slowly shifting regional patterns. From [59, Fig. 7]. Reproduced by permission. 
standard deviations which can be presented for use in diagnosis (cf. [32]). The "profile" so noted does not, for all the reasons given above, represent a true shape comparison between normal and observed or over successive stages of ontogeny. We cannot analyze Thompson's grids in general by studying the displacements of all the grid intersections from one fixed intersection-to do so would be to lose all the variation of the fixcd intersection, to misapprehend all the factors of systematic variation in which that fixed point is implicated. The vector techniques of orthodontia may suggest uses for orthodontic appliances, but they do not aid us in the general mathematical analysis.

\section{MULTIVARIATE MORPHOMETRICS}

One might imagine that a method for measuring shape could be adapted easily to measure shape change, and would thereby automatically make a contribution to the study of transformations. This is simply not true. In terms of measures of shape, however subtle, shape change can only be an abstract vector of differences, one for each measure. This series is in no way the unique mathematical object which Thompson steadfastly envisioned. The landmarks and positions suitable for characterizing shape are likewise not necessarily suited to the characterization of change-we may need to know where everything is going, the relative motions of intercalated points as well as landmarks.

Nor are the subtleties of hiological correlation between separate changes in parts, which is the wellspring of Thompson's method, expressed adequately in variance-covariance matrices. All multivariate techniques, however resourceful, are restricted to linear relations among the variates; but the reality of variation in form is far from linear-it is functional. Pearsonian correlations between characters, which underlie the canonical styles of variation that a multivariate analysis finds, are but a hint of the essential factors underlying the observed transformations of form.

Of all those working in the new multivariate morphometrics, the only one, to my knowledge, who acknowledges these limits is Oxnard. In his collected thoughts on the analysis of form [40], he takes pains to verify statistical insights by comparison with simple biomechanical demonstrations about the observed regimes of variation. All others-for reviews see [3, $11,19,26]$-identify the variation of empirically derived canonical linear combinations with the biological mechanisms producing the variation. This tradition could not be more divergent from the geometric insights of Thompson's scheme. In the reviews I just cited, totaling some four hundred pages, I could not find a single biological drawing, a single visual example of data, to borrow for this essay. The geometry is drained so thoroughly by the formalism of abstract measurement spaces that in most analyses the findings-canonical forms of variation, extreme types, multivariate 
means - cannot be depicted at all, for the requisite point configurations do not satisfy the rules of the three-dimensional Euclidean space in which we and our subjects of study all live. The multivariate techniques capture shape in a way which necessarily misrepresents shape change. The spokesmen for this school tend to invoke Thompson's name (Gould and Johnston, for instance, describe his vision as "multivariate"), but they are studying variation of their indices only, not of the underlying shapes.

This critique applies, it seems to me, whether the measures of shape be themselves classical or modern. The classical approach is best represented by conventional craniometrics, the practitioners of which industriously measure the relative positions and sizes of arbitrarily many landmarks all over the skull or other form. It is becoming recognized that there is an appalling inelegance and redundancy in this method, that data which have been measured as

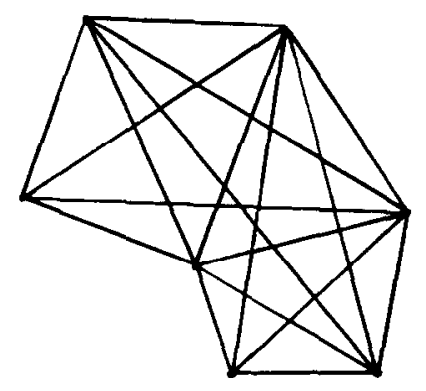

ought to be somehow captured in the form of

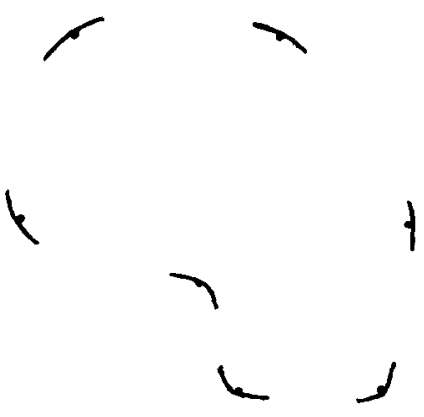

instead. There are modern techniques of outline representation which more parsimoniously capture outlines by successive point coordinates or by some continuous function of polar angle or arc length. These functions are then 
reduced to parameter values by Fourier analysis, direct sampling, or some other construction. I review these developments in [4]. In addition to an assortment of statistical difficulties, they all have the same fallacy as the conventional method: from numerical changes in the parameters, whether amalgamated multivariately or not, it is just not possible to model systematic transformation of the original geometric forms.

At the conclusion of my survey to 1976 of the literature on Cartesian transformations, I find no innovation indigenous to morphometrics or borrowed, no methodological advance for particular styles of data, that is comparable in stature with Thompson's original method. Six decades after its publication the method still resists quantification except in special cases. It remains a fascinating puzzle for biostatistics and mathematical biology, endlessly suggestive and promising, but much more difficult than it was supposed to be. There is yet no progress. Anyone trying to make new headway must begin to build, as I do, exactly where Thompson left off.

\section{THE METHOD OF BIORTHOGONAL GRIDS}

Like several of the authors reviewed, I too have been tantalized enough by the recalcitrant elegance of the Thompson problem to attempt a fresh mathematical unfolding. There are variously detailed presentations of my findings in [4-6]. Here I shall only outline the new responses to old difficulties.

\section{A PRIVILEGED COORDINATE SYSTEM}

My mathematical researches began with an aspect of the Thompson method not previously noted: that the features of a grid as we apprehend them depend capriciously on the grid with which we begin. Consider, for example, the transformation of a square into a $60^{\circ}$ rhombus, drawn twice in Fig. 10. The upper engridment would be described as a vertical shrink by the factor $\sqrt{3} / 2$, the horizontal unchanged, followed by $30^{\circ}$ of shear. In the lower engridment there is instead no shear, but one axis shrinks by $\sqrt{2} / 2$ while the other expands by $\sqrt{6} / 2$. There is no contradiction between these two reports. Any description varies with the starting grid, just as the matrix for a linear transformation changes contravariantly when a vector basis is altered.

I suggest that the lower of the two representations in Fig. 10 is to be preferred. For affine transformations, those which take parallel lines into parallel lines, such a pair of axes is guaranteed. There will always exist a pair of directions, the principal axes of the transform, which are perpendicular both before and after transformation, and with respect to which the observed shape change is described by a pair of dilatations (length multiplications, stretch or shrink), one along each axis. A third parameter fixes the direction of these axes in the organic tissue. 

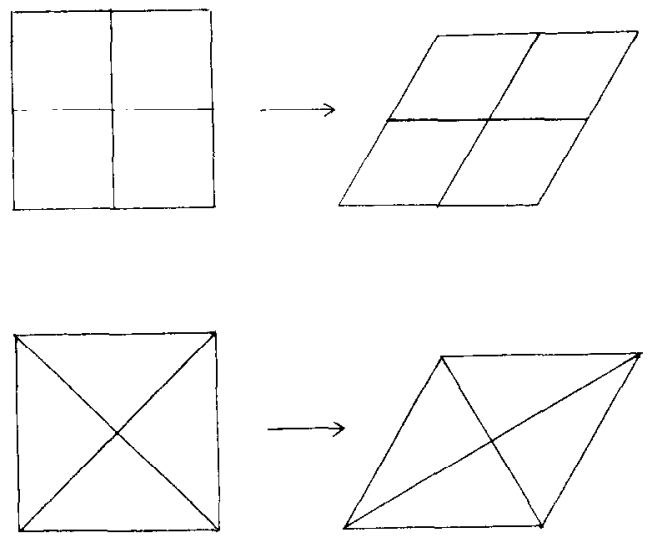

FIG. 10. Two reports of the same affine transformation. Top: Axes do not change in length, but their angle is altered from $90^{\circ}$ to $60^{\circ}$. Bottom: One axis shrinks, the other expands; their angle remains at $90^{\circ}$.
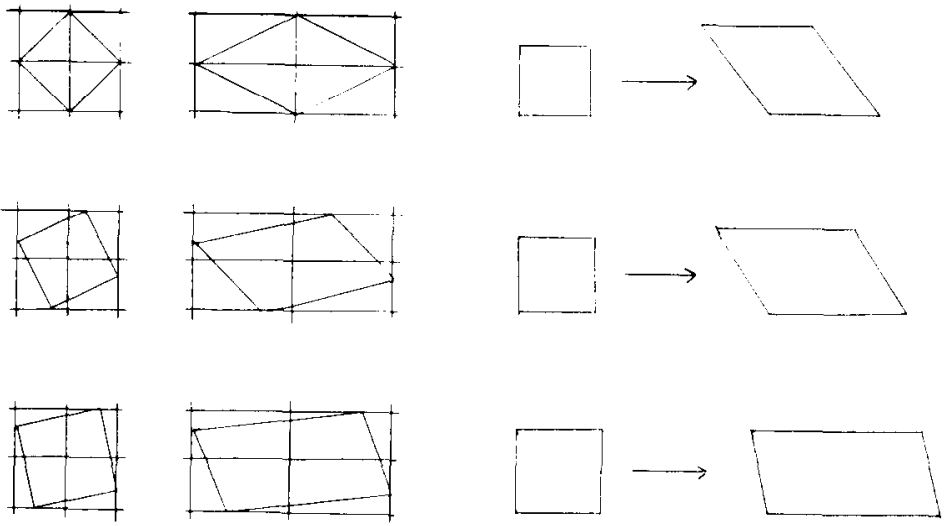

Fig. 11. The same shape change, a 2:1 stretch, attached to a square in three different ways. As the orientation of the axes is altered, the visual impact of the affine transform varies in a complex manner.

In Thompson's original technique, the starting grid is aligned with some feature of the anatomy, the single shape. I would align instead with the shape change itself, regardless of the initial form, which just cuts out a piece of grid after the fashion of a cookie-cutter. In Fig. 11, for example, is shown a single transformation, with dilatations of 2 and 1 , repeated three times. As the orientation of the initial square is varied, the reports of side stretches and shear, and the visual impressions of the right-hand engridments, change 
in a complex manner; but the report using principal axes shows, of course, two parameters constant, only one changing.

Using these axes we can recognize iterations and inverses of a fixed transform with ease. The transformation shown in Fig. 12 is the result of applying that of Fig. 10 twice in succession. This is not at all obvious in the Thompson engridment, but using the representation by principal axes we can tell, for the axes of Fig. 12 are the same as those of Fig. 10, and the dilatations of the latter $(0.5,1.5)$ are just the squares of the dilatations of the former. Likewise, that the transforms of Fig. 13 are inverses is not at all obvious from the Thompson engridment; but we see from the principal-axis representation that the one has dilatations $0.7,2.85$, the other $0.7^{-1}, 2.85^{-1}$, and that the axes are the same.

For more general transformations than the affine we cannot obtain two families of straight lines at $90^{\circ}$ in both images. Still, we may proceed to a privileged coordinate system by a descent to infinitesimals. Consider the projection transform, Fig. 14(a), which maps a window onto its oblique shadow. Through almost every point of the square pass exactly two perpendicular lines mapped into lines perpendicular in the trapezoid, as in Fig. 14(b). The orientation of these lines rotates from point to point of the shapes. We can draw out a collection of smooth curves which are tangent everywhere to one of the local principal axes. Then any two curves are perpendicular wherever they intersect in either image. We cannot draw curves through all points, so we select from the two families to suggest their changing directions by a curvilinear grid. There results the diagram in Figure 14(c).
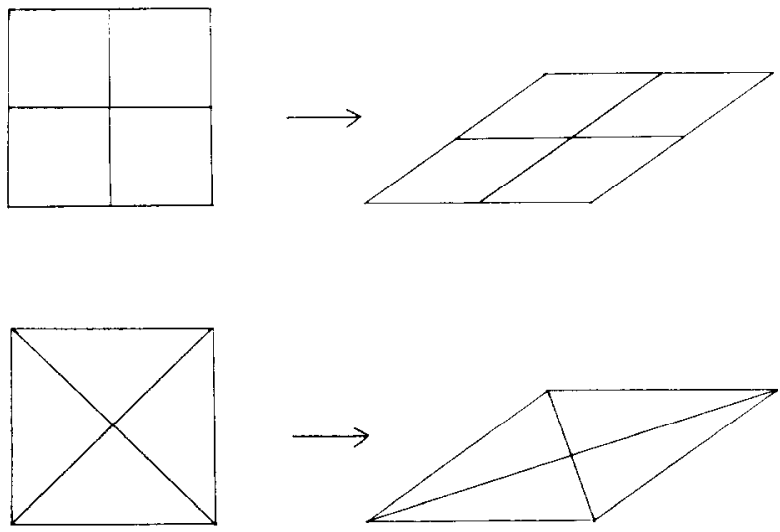

FIG. 12. Two engridments of the transformation which is the twofold iteration of that in Fig. 10. Top: arbitrary axes; bottom: principal axes. 

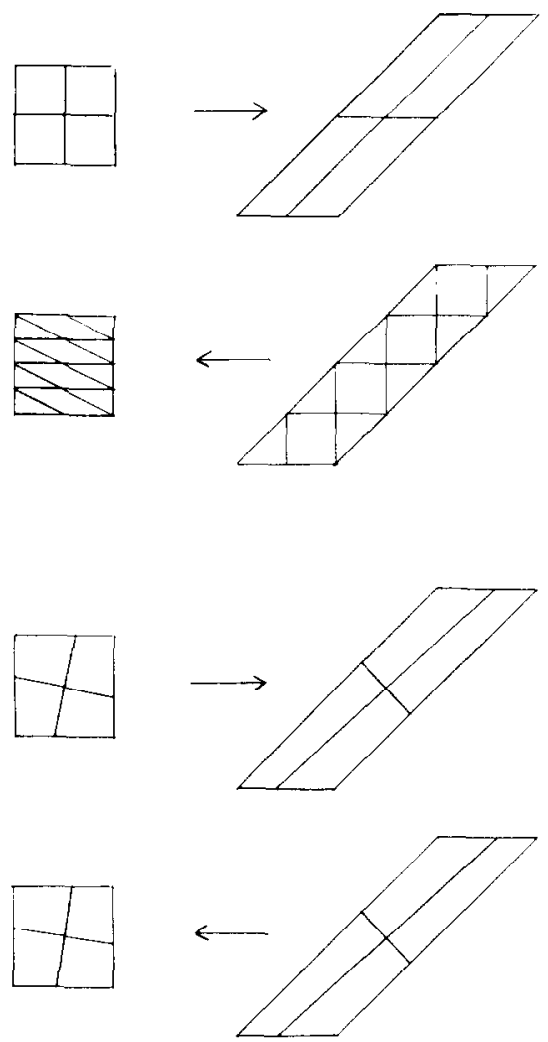

FIg. 13. Two engridments of a transformation and its inverse. Top: arbitrary axes; hottom: principal axes.

The curves do not have a biological reality as loci-they are but threads along which are strung little right angles, tangents and normals. These perpendiculars, I would argue, have a biological meaning. In the small, little squares of sides aligned with these axes, and only these, grow into little rectangles similarly aligned. All other squares grow into oblique parallelograms instead. The observed growth with respect to these axes is symmetric, in other words, and is described by a simple pair of local dilatations. If the axes appear to rotate on the page, it is because of growth in other parts of the beast. The little box is just expanding at two rates in two directions, and knows of no rotations.

My proposal is that these local directions are to be suspected of biological invariance. Any $90^{\circ}$ angle remaining at $90^{\circ}$ through any finite transform is presumed to remain at $90^{\circ}$ through all intermediate stages of the trans- 

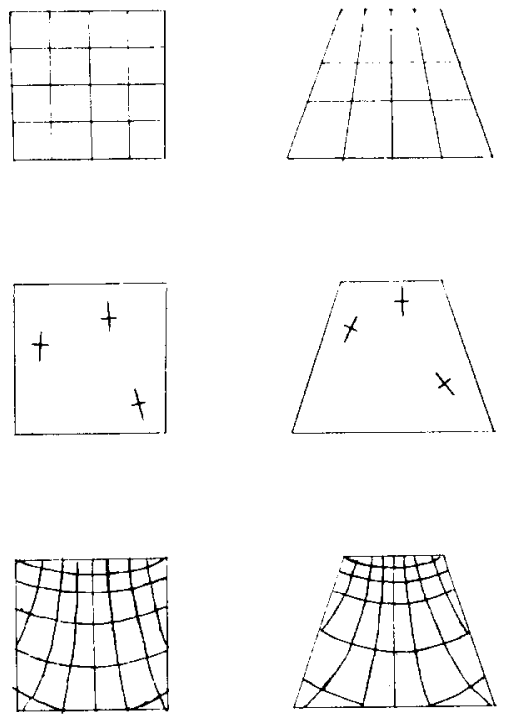

Fig. 14. Top: A projection from a window to its shadow, in arbitrary axes. Middle: Through each point of the correspondence there is one pair of lines which start and finish at $90^{\circ}$ to each other. Bottom: The transform may be represented by the integral curves of these local perpendiculars.

form and into the foreseeable future. All apparent curving and bending of form is produced, on this assumption, by differential growth along axes variously oriented but fixed, along with their right-angled intersections, as by India ink in the tissues. Parts rotate only when they are pushed.

Such a postulate clearly distinguishes the mathematical model from the actual processes underlying shape change. "Growth" here means only adjustment of outline, for all other types of information are excluded from the data base. The curved "lines of growth" are mathematical artifacts, neither anatomical features nor causal agents. In ontogeny, shape change is a response to many different fundamental processes: differentiation, differential division or death, change of cell shape or bulk material parameters, morphogenetic movements. My mathematical model attends to none of these known processes, but only to the resulting geometry. In the comparisons we might make which are not ontogenetic (of individual with norm, of siblings, of endpoints of evolutionary lines), we are even farther from process, dealing instead with evanescent traces of actual ontogenetic controls. It is necessary to assume that points of homologous pairs arise from identical Anlagen and differ only in the quantitative details of the dilatations which brought them to their measured locations. This stance of stubborn geometric reductionism is necessary in view of the great logical 
difficulty of comparing whole shapes in the absence of a complete material history. When the postulate of invariance of axes conflicts with observation, we must go with the data, must say that the transformation has evidently changed. Otherwise we make the simplest possible assumption, that things which have appeared unchanging (the lines of growth, intersecting consistently at $90^{\circ}$ ) will continue so.

To extract information about growth, we compare shapes of matching little cells in a grid and its transformed image. In any computable grid at finite spacing, these cells will be not quite rectangles; in the limit of infinitesimals, they are exact rectangles. For the projective transform, Fig. 14(c), we construe the splaying of the originally square vertical sides as a consequence of excess of horizontal dilatation over vertical. The sides have been pushed out; we feel the axes bracing themselves against their perpendiculars in order to exert the appropriate stress. (The shears of Thompson's engridments contrariwise lead one to a metaphor of stresses externally imposed, a vision less useful.) We read dilatations directly from the diagram pair as ratios of grid spacings in the two images. The convergence or divergence of curves for one shape separately has no particular meaning.

This method is perfectly general. Through almost every point of a differentiable transformation pass two differentials which are at $90^{\circ}$ both before and after transformation. The integral curves of these differentials form a grid whose intersections are at $90^{\circ}$ in both images. These are called the biorthogonal grids for the transformation, for there are two of them, one in each image, corresponding curve for curve, intersection for intersection. We quantify shape change by extracting the two dilatations at every point of either shape, measured along the local canonical axes. Thompson's fundamental error was the construction of diagram pairs which were unsymmetrically specified: rectangular grid on one side, unrestricted grid on the other. In view of the symmetry of all growth in the small, the appropriate grids for any two shapes have the same formal property, that all grid intersections are at $90^{\circ}$. This characterizes the biorthogonal pair uniquely. We have thereby produced a canonical coordinate system which reduces all change of shape to gradients of differential directional growth, without shear. As with the affine transform (Fig. 10 lower), we have specified a coordinate system for the change itself. It becomes possible to measure shape change, then, without measuring shape at all. In fact, the measurement of a particular shape is subsumed under this rubric as the transformation from that shape to some predefined norm.

The method of biorthogonal grids cuts through several of the methodological difficulties common to its predecessors. First, with passive rotation removed, growth is represented by those two dilatations with orientation, in effect a symmetric tensor field over one whole image which can be compared with others on the same image. It is registered everywhere. There is 
therefore no need to register the images in advance. The only privileged points are the landmarks which we use to calculate the one-one correspondence; they happen to align the grid but are not discernible as special points within it. In principle the results of any of the existing schemes for extraction of quantity - growth gradients, vector displacements, multivariate morphometrics- can be reconstructed from a collection of biorthogonal grids, and their biases owing to registration and orientation can even be analytically derived. Second, the technique prescribes explicitly the distances, directions, and gradients that are worth examining. This is managed by abstracting away two particular empirical possibilities, the "moving forms" of Enlow and the tears and morphogenetic movements of Moorrees or Jacobson and Gordon. In the absence of these more precise models of actual change, in which landmarks do not stay landmarks and points arbitrarily close together end up far apart, the proper mathematical model is that of a diffeomorphism, from which the dilatations and principal axes follow rigorously. This is precisely Thompson's own assumption, and so this quantification answers precisely to the needs of his method.

\section{EXISTENCE AND FORM OF BIORTHOGONAL GRIDS}

I now demonstrate formally the existence of biorthogonal grids for arbitrary Thompson transformations. Let $u(x, y)$ be the $x$-coordinate of the image point corresponding to original point $(x, y)$, and $v(x, y)$ likewise the $y$-coordinate. The Thompson transform is then the map $(x, y) \rightarrow$ $(u(x, y), v(x, y))$ taking a region of the $(x, y)$-plane onto a region of the $(u, v)$-plane. Assume the existence of derivatives

$$
\begin{array}{ll}
u_{1}=\frac{\partial u}{\partial x}, & u_{2}=\frac{\partial u}{\partial y}, \\
u_{11}=\frac{\partial^{2} u}{\partial x^{2}}, & u_{12}=\frac{\partial^{2} u}{\partial x \partial y}, \quad u_{22}=\frac{\partial^{2} u}{\partial y^{2}},
\end{array}
$$

and likewise $v_{1}, v_{2}, v_{11}, v_{12}, v_{22}$.

At any point of the $(x, y)$-plane the Jacobian matrix

$$
J=\left(\begin{array}{ll}
u_{1} & v_{1} \\
u_{2} & v_{2}
\end{array}\right)
$$

is the linear transformation taking a vector $(d x, d y)$ in the tangent space about the point $(x, y)$ into a vector $(d x, d y) J=\left(u_{1} d x+u_{2} d y, v_{1} d x+v_{2} d y\right)$ in the tangent space about $(u(x, y), v(x, y))$.

We are interested in the existence of direction pairs which are perpendicular in both tangent spaces. In the tangent space about $(x, y)$, any pair of perpendicular vectors can be written either in the form $(1,0),(0,1)$ or the 
form $(z, 1),(-1, z)$ for some real $z$. We seek a pair perpendicular in both tangent spaces: this means that $(z, 1) J$ must be perpendicular to $(-1, z) J$. That is, $\left(z u_{1}+u_{2}, z v_{1}+v_{2}\right)$ must he perpendicular to $\left(-u_{1}+z u_{2},-v_{1}+z v_{2}\right)$. Taking the dot product, we have

$$
\begin{aligned}
0 & =\left(z u_{1}+u_{2}\right)\left(-u_{1}+z u_{2}\right)+\left(z v_{1}+v_{2}\right)\left(-v_{1}+z v_{2}\right) \\
& =A z^{2}+B z-A,
\end{aligned}
$$

with

$$
\begin{aligned}
& A=u_{1} u_{2}+v_{1} v_{2}, \\
& B=u_{2}^{2}-u_{1}^{2}+v_{2}^{2}-v_{1}^{2} .
\end{aligned}
$$

Whenever $A$ is not zero the discriminant $B^{2}+4 A^{2}$ of the quadratic (1) is positive, implying that there are two real roots $z$ of the equation. This does not mean there are two pairs of perpendicular vectors. The product of the two roots is $-A / A=-1$. Then if one vector is $(z, 1)$, the other is $(-1 / z, 1)$, parallel to $(-1, z)$, representing not another solution but rather the other element of the perpendicular pair. Thus there is only the unique biorthogonal pair of which I spoke. This finding is at least as old as [56].

When $A=0$ but $B \neq 0$ there is one root $z=0$, corresponding to the vector $(0,1)$. This is perpendicular to $(1,0)$ in the $(x, y)$ tangent space, while their transforms $(0,1) J=\left(u_{2}, v_{2}\right),(1,0) J=\left(u_{1}, v_{1}\right)$ are perpendicular in the $(u, v)$ tangent space, for the form $A$ is just their dot product.

There remains the case $A=0, B=0$. Write the latter constraint in the form $u_{1}^{2}+v_{1}^{2}=u_{2}^{2}+v_{2}^{2}=a^{2}$, say, and write $u_{1}=a \cos \theta_{1}, v_{1}=a \sin \theta_{1}, u_{2}=-$ $a \sin \theta_{2}, v_{2}=a \cos \theta_{2}$ for $\theta_{1}, \theta_{2}$ to be determined. Then $0=A=u_{1} u_{2}+v_{1} v_{2}=$ $a^{2}\left(-\cos \theta_{1} \sin \theta_{2}+\sin \theta_{1} \cos \theta_{2}\right)=a^{2} \sin \left(\theta_{1}-\theta_{2}\right)$, so $\theta_{1}=\theta_{2}$ or $\theta_{1}=\theta_{2}+\pi$. Then $J$ is of the form

$$
\left(\begin{array}{cc}
a \cos \theta & a \sin \theta \\
-a \sin \theta & a \cos \theta
\end{array}\right) \text { or }\left(\begin{array}{cc}
a \cos \theta & a \sin \theta \\
a \sin \theta & -a \cos \theta
\end{array}\right)
$$

either a similitude or a similitude followed by the reflection $\left(\begin{array}{rr}1 & 0 \\ 0 & -1\end{array}\right)$. In either case, any pair of vectors perpendicular in the $(x, y)$ tangent space is also perpendicular in the $(u, v)$ tangent space.

In general, the conditions $A=0$ and $B=0$ each specify curved loci in the $(x, y)$-plane, and points where $A=B=0$ are intersections of curves, hence isolated points. Examples can surely be constructed where the constraints $A=B=0$ are in force along extended loci, but their probability of emerging from actual data is vanishingly small. In the language of the theory of flows, the case of isolated points is "generic." Elsewhere than at such points, the 
equation

$$
A d y^{2}+B d x d y-A d x^{2}=0
$$

specifies a pair of differential fields whose integral curves exist and are everywhere extensible, by the usual existence theorems. At each point the two values of $d y / d x$ have product -1 and thus represent perpendicular directions. The whole biorthogonal grid is specified by the single differential equation (2) whose coefficients are forms in the empirical Thompson map given by (1).

It is of interest to examine the behavior of solutions of this system around singularities where $A=B=0$. At such a point, which without loss of generality we may assume to be $(0,0)$ in the $(x, y)$-plane, $J$ may be taken as the identity matrix of order 2 by a suitable change of coordinates in the $(u, v)$-plane. We wish to study the variation of directions $d y / d x$ of our field with displacement from $(0,0)$ in a small neighborhood. Write $(x, y)=$ $(e \cos \theta, e \sin \theta)$, where $e$ is small. Then to first order in $e$,

$$
\begin{aligned}
& u_{1 \mid(x, y)}=1+e \cos \theta u_{11}+e \sin \theta u_{12}, \\
& u_{2 \mid(x, y)}=\quad e \cos \theta u_{12}+e \sin \theta u_{22}, \\
& v_{1 \mid(x, y)}=\quad e \cos \theta v_{11}+e \sin \theta v_{12}, \\
& v_{2 \mid(x, y)}=1+e \cos \theta v_{12}+e \sin \theta v_{22} .
\end{aligned}
$$

Likewise to first order,

$$
A_{(x, y)}=u_{1} u_{2}+v_{1} v_{2}=e\left[\cos \theta\left(v_{11}+u_{12}\right)+\sin \theta\left(v_{12}+u_{22}\right)\right],
$$

and

$$
B_{(x, y)}=2 e\left[\cos \theta\left(u_{11}-v_{12}\right)+\sin \theta\left(u_{12}-v_{22}\right)\right]
$$

The coefficients of the forms $A$ and $B$ are all zero only in very unlikely circumstances, for instance, if $u$ and $v$ form a pair of conjugate harmonic functions. For all empirical data sets we may assume that the first-order terms are not all zero near the singularity $(0,0)$ and that they govern the behavior of the solutions there.

Let us write $L=v_{1}+u_{2}, M=u_{1}-v_{2}$, and let $L_{1}, M_{1}$ be their partial derivatives with respect to $x$ at $(0,0)$ and $L_{2}, M_{2}$ their partials with respect to $y$ there. Then we have

$$
\begin{aligned}
& A=e\left(L_{1} \cos \theta+L_{2} \sin \theta\right)=e \cos \theta\left(L_{1}+L_{2} \tan \theta\right), \\
& B=2 e\left(M_{1} \cos \theta+M_{2} \sin \theta\right)=2 e \cos \theta\left(M_{1}+M_{2} \tan \theta\right) .
\end{aligned}
$$


When we substitute these values in the equation (2) describing the biorthogonal field near $(0,0)$, and divide through by $e \cos \theta$, we arrive at

$$
\left(L_{1}+L_{2} \tan \theta\right) z^{2}+2\left(M_{1}+M_{2} \tan \theta\right) z=L_{1}+L_{2} \tan \theta,
$$

where $z=d y / d x$. Note that the variable $e$ has dropped out-to first order, the biorthogonal directions are independent of $e$ for $e$ near zero.

Consider those rays $\theta=\theta^{*}$ out of the singularity $(0,0)$ which lie along one or the other of the perpendicular directions at every point upon them. If the vector $(1, z)$ is on the ray $\theta=\theta^{*}$, then $z=\tan \theta^{*}$. Substituting this value in (3), we have as the equation for the azimuth $\theta^{*}$ of these special rays

$$
\left(L_{1}+L_{2} \tan \theta^{*}\right) \tan ^{2} \theta^{*}+2\left(M_{1}+M_{2} \tan \theta^{*}\right)=L_{1}+L_{2} \tan \theta^{*}
$$

If $L_{2} \neq 0$ this is a cubic equation in $\tan \theta^{*}$. (If $L_{2}=0$ we may still interpret it as a cubic with one root $\tan \theta^{*}=\infty, \theta^{*}= \pm \pi / 2$.) The cubic has either one real root or three. In the latter case we may assume all roots distinct-the "generic" case, coincident roots occurring only in data with probability zero. For each value of $\tan \theta^{*}$, of course, therc are two directions, exactly opposite each other, which are aligned with the biorthogonal pairs at all their points.

The two possibilities occur as the solution curves away from the singularity are concave or convex to the singularity. (A simple geometric argument shows they cannot be mixed.) The grids then appear as follows:

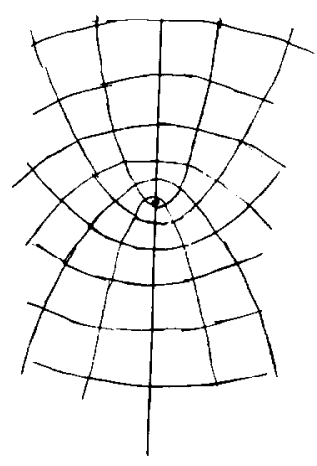

One root.

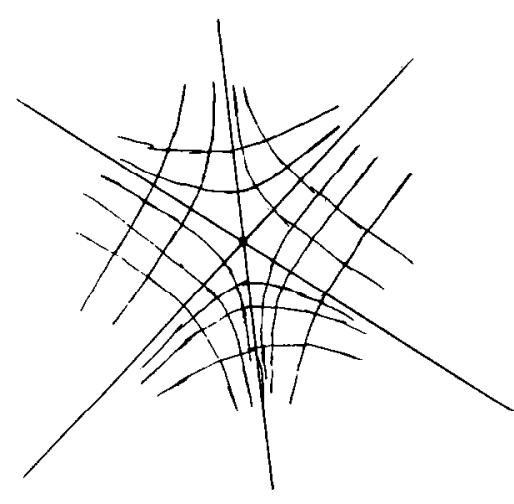

Three roots. 
These correspond to fields of index -1 and +1 , respectively, as described in [22, pp. 30-34]. In empirical analyses, either of these occurs from time to time.

At all points other than these singularities the appearance of biorthogonal grids in a small neighborhood is that of a regular network, a so-called "orthogonal parametrization," the distorted image of regular graph paper, as in Figs. 15 or 17 below; cf. [8, p. 183, Corollary 2; p. 187, Problem 9]. Furthermore, all coordinate curves of a typical grid begin and end only at the boundaries of the forms. For if they did not, they would have to either end at a singularity of the system or cycle around a singularity in a spiral or a loop; and the two generic types of singularities do not allow this behavior.

\section{SAMPLE COMPUTATIONS}

In computing these curve systems it is necessary to know in advance the one-one correspondence between the images. It cannot be explicitly input to a computer, but must be inferred from a sample of corresponding points by some general interpolation routine. Matters are simplest if one assumes no data upon the interiors of the forms, but only a boundary correspondence. I have in fact adopted the following strategy. The data for a computed correspondence between two images are discrete pairs of homologous landmarks, the corners of the quadrilaterals in Fig. 15 or the seven specific anatomical locations of Figs. 16 and 17. These landmarks determine polygonal boundaries for each image, along corresponding sides of which the homology is presumed linear. Let one image now be fixed as the domain of the coordinate transformation. Then the $x$ - and $y$-coordinates of the mapping function are separate solutions of the Dirichlet problems $\nabla^{2} x=0$, $\nabla 3 y=0$ on that image with boundary values assigned equal to the real and imaginary parts, respectively, of the homologous boundary points on the other (the target) image. The resulting correspondence, engridded traditionally in Fig. 16, is smooth and intuitively pleasing. Further methodological development is needed to take into account interior data points.

Any algorithm approximately solving the Dirichlet problem can be modified to approximate segment by segment the integral curves of the field of little perpendiculars. The whole procedure I call the method of biorthogonal grids. I have constructed a preliminary computcr program which handles the two-dimensional version in which biological shapes are represented by outline drawings.

In practice, the grids appear to be aligned in very sensible ways. Consider, for instance, the transform in Fig. 15, the growth of an abstract "foot." The input data consisted of the quadrilateral corners, coordinate by coordinate; the output is the mesh filling the forms, two grids which correspond point for point between the images. At right I have enlarged the upper grid and written in selected dilatations for the "growth" from square 
$<$

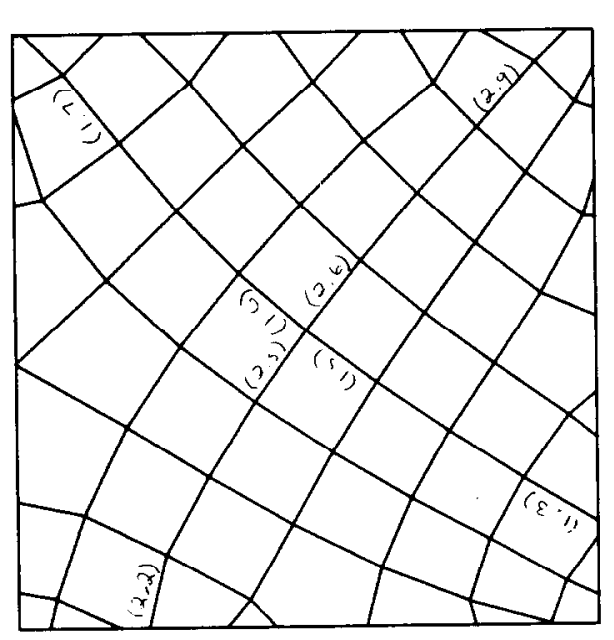

a

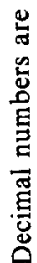

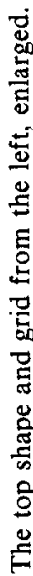

芦竞

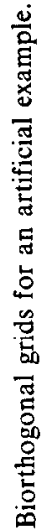

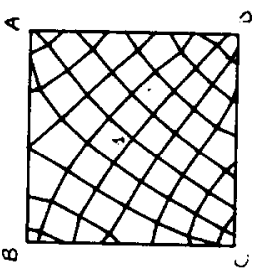

is

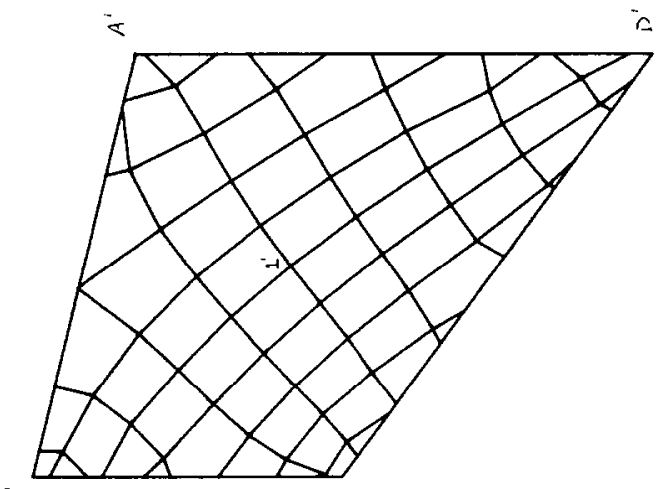

is 
to quadrilateral. A report of distances and angles between the corners, while encapsulating the actual shape change, would be tedious and would not isolate "factors" in any useful sense. The biorthogonal analysis is much more suggestive. Line BD is close to the major axis of growth, which is bowed slightly above it, with dilatations graded from 2.2 at one end to 2.9 at the other. Perpendicular to this, and also nearly aligned with a diagonal of the original square, is a minor axis close to $\mathrm{AC}$ with dilatations graded from 1.3 near $\mathrm{C}$ to 1.7 near $\mathrm{A}$. The remaining axes and dilatations intergrade nicely. By means of the biorthogonal display we have been able to summarize this entire transform in but two main gradient-bearing curves. There is no need to report changes of angle. There is only change of scale, continuously varying in alignment and magnitude. The change is encapsulated in the derived coordinate system; the original configuration of landmarks, their relative distances and angles, is quite accidental.

It is interesting to apply this method to the problem of hominization, Cartesian grids for which have been drawn previously by Thompson (cf. Fig. 2), Hutchinson [23, p. 600], Kummer [28, p. 111], and Sneath [51, pp. $67,98]$. Here I shall explain the grid of the transformation from chimpanzee to modern man. Intermediates of the progression are more fully explored, and an interesting orthogenesis unearthed, in Bookstein [6].

Before any computation it is necessary to have fixed a suitable plane projection and reliable landmarks whose relative motions fairly summarize the one-one correspondence between the two forms. I selected the familiar midsagittal plane (about which the skull is symmetric) and seven landmarks upon it that I could locate from drawings: the first upper incisor, I; the nasion or bridge of the nose, $\mathrm{N}$; the bregma, $\mathrm{B}$; the lambda, $\mathrm{L}$; the inion or occipital protuberance, $\mathrm{O}$; the external auditory meatus, $\mathrm{A}$; and the last upper molar, M. I noted the coordinates of these points for the representative "modern European" in Fig. 177 of [9] and for the female chimpanzee in Fig. 79 of [12]. Although the heptagon formed by the landmarks systematically misses the large convexities of the outline, the deficit shapes are similar in the two images, so that not much error is introduced thereby. Smaller features, such as the orbital ridge and the mastoid process, are not fairly represented by the sevenfold pairing, but their misrepresentation does not alter the general picture.

The Cartesian grid for this transformation, with the interpolation computed as I have described, is presented in Fig. 16. It closely resembles the grids published elsewhere both in its general lineaments and in its shifting inscrutable skein of changes of spacing and orientation. The biorthogonal grid, drawn in Fig. 17, is considerably different. Just as two axes summed up Fig. 15, so do three axes, I believe, summarize the shape change of Fig. 17. I shall denote these axes by the numbers of the arrows pointing to their ends in the bottom diagram: axes 1-5, 2-4, and 3-6. Some dilatations along 


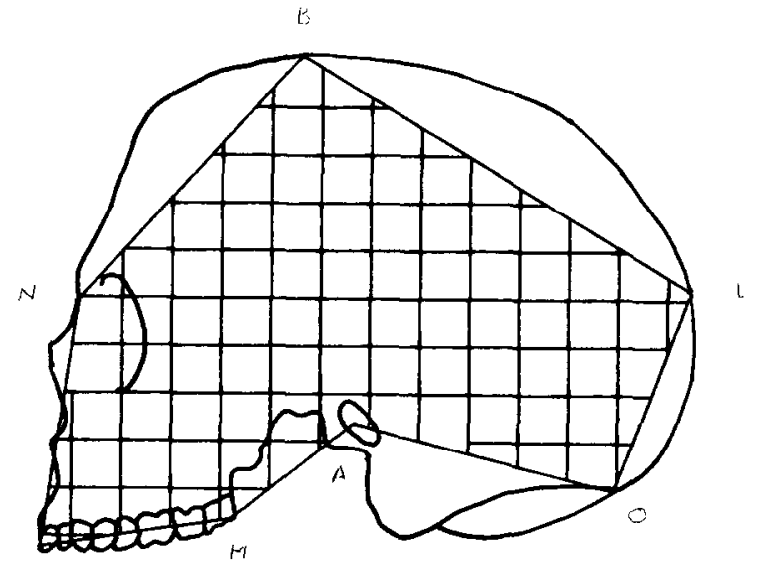

I

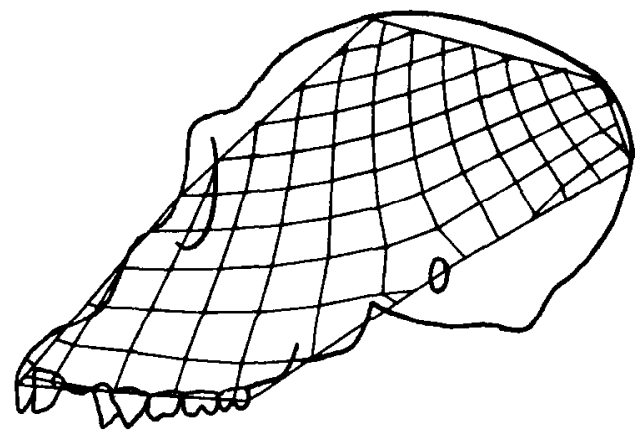

FIG. 16. Thompson-style grid for the transform from Homo sapiens to chimpanzee, with biological boundary superimposed. The letters identify landmarks named in the text.

these axes and elsewhere are written in upon segments of the grid for modern man. They represent relative growth from chimpanzee to modern man of the segments they bestride.

Along its length the axis 3-6 participates in two processes usually reported separately. The dilatations along its lower reaches are less than unity, in accordance with the shrinkage in length of the midface and the closure of the diastema. At its upper end, near arrow 3, the dilatations are considerably greater than unity. Together they constitute a growth gradient which is really quite steep. Two features notably exclude this axis from the purview of conventional craniometrics. It is virtually straight in the chimpanzee outline but strongly bent, owing to growth elsewhere, in the modern form; and it represents a mixed allometry, partly positive, partly negative, so that the arc-length change from end to end is smaller than the changes of 


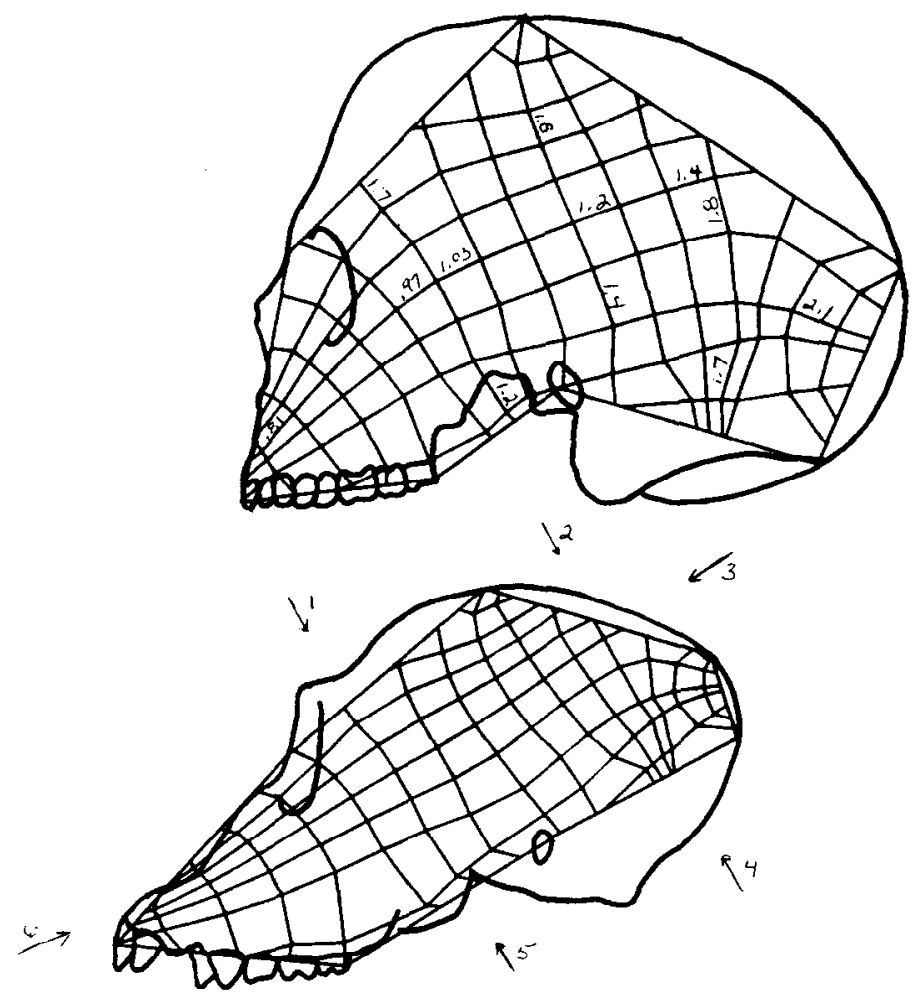

Fig. 17. Biorthogonal grids for the same transform. The decimal numbers are selected dilatations. The three main axes of change, indicated by arrows, are described in the text.

either of its subarcs taken separately. Of course, as the place where the change occurs is not a landmark, the subarcs could not routinely be measured separately in any case.

The curve 3-6 is not necessarily a strict optimum for any of the aspects I just reported. I could as well have selected one of the curves "parallel" to it on either side or some other curve, even nearer, that the computer happened not to draw. The curve 3-6 merely typifies a systematic variation along its length of direction and dilatation throughout its corridor of the diagram.

Perpendicular to this major axis 3-6 are two other axes worth examining. Axis 1-5 bears another monotone gradient, from 1.24 at its lower end to 1.7 at its upper. It represents the pushing-out of the orbit from the cranial base, increasing the space available for forebrain at the same time the face is shrinking. Axis 2-4 parallels 1-5 near the back of the head. The dilatations along it are virtually constant between 1.7 and 1.8; it represents an even 
vertical expansion of the hind braincase at a rate greater than the longitudinal expansion along the major axis: a directionality of expansion which, of course, gives increased volume with the least increase in weight.

In this manner the observed shape change, chimpanzee to human, is described by a smoothly varying pattern of oriented dilatations instead of a congeries of arbitrary craniometric indices. If angles do change, it is because of differential growth elsewhere in the image. The axes are fixed at $90^{\circ}$; there is no rotation about the cranial base or anywhere else. Rather, the back of the head is pushed down-and the orbit pushed out-by an axis of growth perpendicular to the major one. The whole summary of the shape change is surprisingly efficient, and makes no explicit use of the landmarks at all. In fact, these axes correspond to none of the conventional landmarks, and the variation they describe so concisely is spread thin among a host of suboptimal indicators throughout the literature on the subject.

\section{A FUTURE FOR TRANSFORMATION ANALYSIS}

I believe the method of biorthogonal grids to be not a compromise like those of Sec. III but rather a basic step toward the direct and automatic quantification of transformations. The only test is by empirical exercise, in the analysis of sequences for seriation or prediction and in the statistical and substantive interpretation of the dilatation patterns extracted. Of course, a great many methodological questions remain to be explored. Two classes are particularly crucial.

1. Computations need to be computer-mounted for more realistic representations of biological shape. A general method needs to be able to handle curved outlines, internal data points, surfaces, and solid forms in three dimensions. These are likely to be difficult technical problems, as they go beyond currently available techniques of computational geometry.

In extending the biorthogonal method to handle three-dimensional data, it is not necessary to alter the mathematical argument in any essentials. In the planar case, the subject of all my examples here, the boundary by itself is not a rigid structure, and its changes in length do not determine its changes in shape. It is not enough to know at what rates it is growing all around, but in which directions as well. Then the biorthogonal engridment is necessarily in terms of differential growth throughout the inside of the form. When we add a dimension we encounter the surfaces of solids, which are curved two-dimensional manifolds. These forms will in general be more or less convex. Now closed strictly convex surfaces are mathematically rigid. Specify geodesic distances between points in the surface and the whole structure is fixed up to a combination of Euclidean reflection, translation, and rotation. (The demonstration of this is known as the Problem of Weyl: cf. [53, Sec. X.6].) If we specify length changes on a 
surface, the ensuing solid form change is fully determined, including all bulging. In short, for three dimensions change in shape is wholly specified by change in surface distances-this was not true for outlines in the plane. Hence our grid, too, can be restricted to the surface itself; we need not construe "pressures from the interior" as in the metaphors of the planar analysis. This is just as well in view of the great practical difficulty of collecting morphometric data from the insides of living biological forms.

In three dimensions, then, as in two, the Thompson problem is effectively one of transformations on surfaces. Now locally any reasonable surface can be mapped conformally onto the plane. (The cartographers discovered this long before the mathematicians; cf. [27, Sec. 58].) In such a map distances may be distorted, but by the same factor in all directions away from any point; then angles are preserved. Two corresponding neighborhoods on two curved surfaces may each be mapped conformally onto planes. The biorthogonal axes through neighborhoods are defined by reference only to angles, and so are unchanged by any such combination of maps. Then the very same mathematical theory of coordinate form applies locally: the distorted grids and the singularities we have already seen are just to be pasted upon the curved forms. One need not even execute the conformal mapping in the course of computation. The formulae are set forth entirely in terms of tangent spaces, precisely so that they might generalize verbatim to surfaces in three-dimensional space.

The only difference between the analysis in two dimensions and the analysis in three is that closed surfaces in space have no boundaries. Coordinate curves of a biorthogonal grid extend indefinitely, winding around and around the form in an ergodic net. Furthermore, no coordinate system without singularities can apply to a simple closed surface anyway, and in particular no biorthogonal system could possibly do so. (This is Poincaré's Theorem; cf. [8], Sec. 4.5, especially p. 282.) Then we must restrict the analysis to more or less local patches of surface, perhaps hemisphere-sized, set at the discretion of the investigator.

To execute a biorthogonal analysis in three dimensions will be straightforward once we have a one-to-one twice differentiable correspondence between empirically measured surfaces. Techniques for the computation of such correspondences in useful form are prologue to the biorthogonal method, not properly part of the technique itself. There seems, however, to be no published algorithm to refer to. I outline a possible approach using Coons surfaces on cylindrical coordinates in [4].

2. Statistical conventions of considerable geometric sophistication are needed to analyze the output of these computations. The representation of transforms locally in terms of two dilatations and an orientation reduces growth in the large to a symmetric tensor field. It must be possible to 
compare these with each other, add them together, "factor" them, average them. It ought to be possible to deal with them as dependent variables to be parceled out and "explained" in analogues of the analysis of variance. For instance, we should be able to fit a series of powers of the same growth field to a given series of shapes in some optimal way, and to refer to the "fitted growth" and the "unexplained growth" for each transformation. We also might want to perform a morphometric analysis of covariance, optimally dividing out some sort of allometric growth before fitting a secular trend. We need, finally, to be able to simulate arbitrary dilatation fields upon arbitrary forms: given a shape, to apply to it any growth that we care to, and then visualize the resulting curving form, thereby in particular extrapolating and interpolating.

With any of these improvements, the method of biorthogonal grids clearly can remedy the presently ungeometrical content of the methods of shape and growth analysis. Several applied problems cry out for a sound morphometrics: besides orthodontia, these include embryology normal and abnormal, computed tomography, plastic surgery, and all the many special sciences of specific organs animal and vegetable. The advantages of a geometric morphometrics are incalculable. It is the ubiquitous unsatisfied demand for such a formalism, a calculus of form, that has kept the Thompson problem in the eye of the profession through sixly years of stasis. A technology for routine diagnosis, analysis, and prediction of shape would spread a fertile new quantification throughout the biosciences.

I acknowledge the support of the Michigan Society of Fellows and of the Statistics Research Laboratory, William Ericson, director; the cooperation of the Department of Statistics and the Division of Biological Sciences; and the counsel of Professors Arnold Kluge, Robert Moyers, Waldo Tobler, and Carl Simon, all of the University of Michigan.

\section{REFERENCES}

1 G. S. Avery Jr., Structure and development of the tobacco leaf, Am. J. Bot. 20, 565 (1933).

2 R. E. Blackith, R. G. Davies, and E. A. Moy, A biometric analysis of development in Dysdercus fasciatus sign, Growth 27, 317 (1963).

3 R. E. Blackith and R. A. Reyment, Multivariate Morphometrics, Academic, New York, 1971.

4 F. L. Bookstein, The measurement of biological shape and shape change, unpublished Ph.D. dissertation, Univ. of Mich., 1977.

5 F. L. Bookstein, The geometry of shape change, submitted for publication.

6 F. L. Bookstein, Orthogenesis of the hominids: an exploration using biorthogonal grids, Science, to appear, 1977.

7 V. Brown and R. G. Davies, Allometric growth in two species of Ectobius, J. Zool. Lond. 166, 97 (1972). 
8 M. P. do Carmo, Differential Geometry of Curves and Surfaces, Prentice-Hall, Englewood Cliffs, N.J., 1976.

9 W. E. le Gros Clark, The Fossil Evidence for Human Evolution, 2nd ed., Univ. of Chicago Press, Chicago, 1964.

10 E. H. Colbert, Siwalik mammals in the American Museum of Natural History, Trans. Am. Philos. Soc. 26, 1 (1935).

11 R. S. Corruccini, Multivariate analysis in biological anthropology: some considerations, J. Hum. Evol. 4, 1 (1975).

12 A. Delattre and R. Fenart, L'Hominisation du Crâne, Ed. du C.N.R.S., Paris, 1960.

13 D. H. Enlow, The Human Face, Harper and Row, New York, 1968.

14 D. H. Enlow, Handbook of Facial Growth, Saunders, Philadelphia, 1975.

15 R. O. Erickson, Relative elemental rates and anisotropy of growth in area: a computer programme, J. Exp. Bot. 17, 390 (1966).

16 S. J. Gould, Allometry and size in ontogeny and phylogeny, Biol. Rev. 41, 587 (1966).

17 S. J. Gould, Evolutionary paleontology and the science of form, Earth-Sci. Rev. 6, 77 (1970).

18 S. J. Gould, D'Arcy Thompson and the science of form, New Lit. Hist. 2, 229 (1971).

19 S. J. Gould and R. F. Johnston, Geographic variation, Ann. Rev. Ecol. Syst. 3, 457 (1972).

20 P. B. Green, Pathways of cellular morphogenesis, J. Cell Biol. 27, 343 (1965).

21 P. B. Green, Cell morphogenesis, Ann. Rev. Plant Physiol. 20, 365 (1969).

22 H. Hopf, Selected Topics in Differential Geometry in the Large, lect. notes by T. S. Klotz, New York Univ., 1955.

23 G. E. Hutchinson, In memoriam, D'Arcy Wentworth Thompson, Am. Sci. 36, 577 (1948).

24 J. S. Huxley, Problems of Relative Growth, Methuen, London, 1932.

25 A. G. Jacobson and R. Gordon, Changes in the shape of the developing vertebrate nervous system analyzed experimentally, mathematically, and by computer simulation, J. Exp. Zool. 197, 191 (1976).

26 C. J. Kowalski, A commentary on the use of multivariate statistical methods in anthropometric research, Am. J. Phys. Anth. 36, 119 (1972).

27 E. Kreyszig, Introduction to Differential Geometry and Riemannian Geometry, Univ. of Toronto Press, Toronto, 1968.

28 B. Kummer, Untersuchungen über eine morphologische Reihe in der Phylogenie des Menschenschädels, Homo 3, 109 (1952).

29 R. S. Lull and S. W. Gray, Growth patterns in the Ceratopsia, Am. J. Sci. 247, 492 (1949).

30 P. B. Medawar, Size, shape, and age, in Essays on Growth and Form (P. B. Medawar and W. E. le Gros Clark, Eds.), Clarendon, Oxford, 1945.

31 P. B. Medawar. D'Arcy Thompson and Growth and Form, in D'Arcy Wentworth Thompson: the Scholar-Naturalist, Oxford Univ. Press, London, 1958.

32 W. W. Merow, Cephalometrics, in [14].

33 C. F. A. Moorrees and L. Lebret, The mesh diagram and cephalometrics, Angle Orthod. 32, 214 (1962).

34 C. F. A. Moorrees and L.Lebret, R. B. Reed, R. L. Kent, Jr., and C. N. Glatky, The computerized mesh diagram analysis, in Transactions of the Third International Orthodontic Congress, Crosby Lockwood Staples, Frogmorc, G.B., 1975.

35 M. L. Moss and L. Salentijn, The logarithmic growth of the human mandible, Acta anat. 77, 341 (1970). 
36 A. E. Needham, On relative growth in Asellus aquaticus, Proc. Zool. Soc. Lond. A 107, 289 (1937).

37 A. E. Needham, On relative growth in Asellus aquaticus. II, Proc. Zool. Soc. Lond. A 113, 44 (1943).

38 A. E. Needham, The form-transformation of the abdomen of the female pea-crab, Proc. Roy. Soc. Lond. B 137, 115 (1950).

39 A. E. Needham, The Growth Process in Animals, Van Nostrand, Princeton, 1964.

40 C. E. Oxnard, Form and Pattern in Human Evolution, Univ. of Chicago Press, Chicago, 1973.

41 D. M. Raup, Geometric analysis of shell coiling, J. Paleontol. 40, 1178 (1966).

$42 \mathrm{O}$. W. Richards, D'Arcy W. Thompson's mathematical transformation and the analysis of growth, Ann. N.Y. Acad. Sci. 63, 456 (1955).

43 O. W. Richards and A. J. Kavanagh, The analysis of growing form, in Essays on Growth and Form (P. B. Medawar and W. J. le Gros Clark, Eds.), Clarendon, Oxford, 1945.

44 O. W. Richards and G. A. Riley, The growth of amphibian larvae illustrated by transformed coordinates, J. Exp. Zool. 77, 159 (1937).

45 R. M. Ricketts, A principle of arcial growth of the mandible, Angle Orthod. 42, 368 (1972).

46 D. da Riva Ricci and B. Kendrick, Computer modelling of hyphal tip growth in fungi, Can. J. Bot. 50, 2455 (1972).

47 P. Salamon, A. List Jr., and P. S. Grenetz, Mathematical analysis of plant growth, Plant Physiol. 51, 635 (1973).

48 O. Schuepp, Wachstum und Zellanordnung im Sprossgipfel, Ber. Schweiz. Bot. Ges. 62, 592 (1952).

49 O. Schüepp, Meristeme, Birkhäuser, Basel, 1966.

$50 \mathrm{~K}$. A. G. Shiclls, Growth of a productid shell and its implication on a method of statistical correlation, Nature 205, 878 (1965).

51 P. H. A. Sneath, Trend-surface analysis of transformation grids, J. Zool. Lond. 151, 65 (1967).

52 R. R. Sokal and P. H. A. Sneath, Principles of Numerical Taxonomy, Freeman, San Francisco, 1963.

53 J. J. Stoker, Differential Geometry, Wiley, New York, 1969.

54 G. Teissier, Relative growth, in The Physiology of Crustacea (T. H. Waterman, Ed.), Academic, New York, 1960.

55 D'A. W. Thompson, On Growth and Form, 1917, 1942; abr. ed. (J. T. Bonner, Ed.) Cambridge U. P., 1961.

56 M. A. Tissot, Memoires sur les representations des surfaces, Gauthier, Paris, 1881.

57 W. R. Tobler, D'Arcy Thompson, and beyond, by computer, unpublished manuscript.

58 G. F. Walker and C. J. Kowalski, A two-dimensional coordinate model for the quantification, description, analysis, prediction, and simulation of craniofacial growth, Growth 35, 191 (1971).

59 G. F. Walker and C. J. Kowalski, A new approach to the analysis of craniofacial morphology and growth, Am. J. Orthod. 61, 221 (1972).

60 F. Yates, The place of statistics in the study of growth and form, Proc. Roy. Soc. Lond. $B$ 137, 479 (1950). 